\title{
SICKNESS ABSENCE OF WOMEN BUS CONDUCTORS IN LONDON TRANSPORT (1953-1957)
}

\author{
BY \\ C. J. CORNWALL and P. A. B. RAFFLE \\ From the London Transport Executive
}

(RECEIVED FOR PUBLICATION MARCH 27, 1961)

\begin{abstract}
The sickness-absence experience of women bus conductors employed by London Transport on their central (red) buses is analysed for the years 1953 to 1957 inclusive. The experience of single and married women is given separately, and only absences lasting four days or more (all of which are supported by medical certificate) are included. Results are given in quinquennial age groups for each of $\mathbf{2 0}$ broad diagnostic groups and comment is made on each group, emphasis being placed on the comparative experiences of single and married women. Both the numbers of spells of absence and the numbers of days of absence are considered. It is found generally that married women conductors have considerably more sickness absence than single women conductors of the same age: this applies both to spells and days of absence. Analysis of the difference by diagnostic group suggests that there is a propensity, of social origin, for married women to have more absences.
\end{abstract}

An account of the principles and methods followed in the compilation of the London Transport sickness-absence statistics was given in Health in Industry (London Transport Executive, 1956). The sickness-absence experience of various groups of London Transport male wages staff (including male conductors) and male and female clerical staff over the period 1949-52 was set out in detail in that publication, analysed by age and by broad diagnostic group. This paper extends that work by giving some similar analyses, but for the subsequent quinquennium, for a group of London Transport female wages staff, namely the women conductors of the central (red) buses.

The sickness-absence experience of single and married women has been separated, and emphasis has been placed on the comparison of the sickness absence of these "single" and "married" women who both follow the same occupation. The subdivision into single and married women is according to whether they are known as "Miss" or "Mrs" respectively; information is not available to the employer enabling the "Mrs" group to be subdivided into women with husbands living and widows.

Recruitment of women conductors started in 1940, was discontinued after the war, recommenced in 1950, and has continued to the present time. Thus the population under consideration consists mainly of women with less than eight years' service but there are some with up to 18 years' service.

"Sickness absence"' is taken to mean absence from work accepted by the employer as attributable to sickness or injury. Medical certificates are required not later than the third day of absence. This investigation has been limited to spells of absence lasting four days or longer, and thus includes only absences supported by a medical certificate. The majority of spells of sickness absence terminate with the employee's recovery and return to her former work. When an absence lasts for six months, however, the recorded absence is curtailed at 182 days, as after that duration of absence, an administrative decision may be taken regarding the retention of the employee's name on the books, or her premature retirement on the grounds of ill health. It should be noted that in all cases, the number of days' absence ascribed to a particular spell of absence is the number of days on the calendar from the first day of absence from work to the day preceding the employee's return to work, both dates inclusive; in other words, the length of a spell of absence in days is recorded on the basis of seven days to the week.

Women conductors are medically examined before appointment and must reach appropriate physical standards. An employee who has been absent for more than four weeks (or in the case of certain 
diagnoses certified by the general practitioner, including accidents, for a shorter period) must be certified fit by a London Transport Medical Officer before resuming duty. When the appropriate standard of health is not reached, the employee may be found suitable alternative employment, but the scope for alternative employment of women in the London Transport Executive's undertaking is very limited.

This investigation covers the five years 1953 to 1957. Sick pay was not paid to women conductors before December 1956. Since then, limited amounts have been payable, after a week of absence, to women conductors who are over age 25 and who have more than five years' service. Many of the married women do not contribute to the National Insurance Scheme, and thus receive no sickness benefit from the State. Women conductors have been eligible for membership of the Sick Benefit Section of a large Friendly Society, which draws its membership mainly from the bus crews, only since March 1957. Thus throughout the majority of the quinquennium under review, women could not obtain sickness benefit from this source.

The detailed results of the investigation are set out in Tables 1 to 21 in the Appendix. In these Tables data are given for single and married women, and subdivided into quinquennial age groups. Sickness absence has been split into 20 broad diagnostic groups, which conform to those given by London Transport Executive (1956), and are based on the three-figure International Statistical Classification of Diseases and Injuries adopted in 1948 by the World Health Organization.

For each diagnostic group, and each quinquennial age group, the following indices have been calculated:

(i) Average annual duration per person, which is the average number of calendar days of sickness absence per employee per annum;

(ii) Annual inception rate (spells), which is the average number of spells commencing in a year per employee;

(iii) Average length of spell, which is the average length in days of each recorded spell of sickness absence.

In addition, the number of "man-years" of exposure to risk, the number of spells of absence commencing in the period, and the number of days of absence during the period are given. It is emphasized that these only relate to spells of four days' duration or more.

Graphs have been drawn to assist in the interpretation of the Tables, with particular reference to the comparison between the sickness-absence experiences of married and single women. There were no single women bus conductors older than 60 , the normal retiring age, and very few married women conductors over that age; the graphs have therefore been curtailed at age 60 . It should also be noted that the exposed-to-risk figures for single women in the 50-54 and the 55-59 age groups and for married women in the 55-59 age group are very small, and consequently little weight should be attached to the results for those groups. The corresponding sections of the graphs are shown as dotted lines.

\section{Broad Diagnostic Groups}

\section{All Diagnostic Groups Combined.-(Table 1).}

Graph 1a and $\mathrm{b}$ show quite clearly that the single women bus conductors experience significantly lighter sickness absence than the married women. This is so at all ages. The excess for married women is of the order of $40 \%$ for spells, and as much as $60 \%$ for days of absence. In each case, the annual inception rate (spells) remains fairly constant, but the average annual duration per person increases steadily with age.

BDG. 1.-Tuberculosis (Table 2) (International Code Nos. 001-019).

There were only seven spells among the single women, compared with 11 amongst the married women. This difference is not significant. No comparison by age is possible with such small numbers. The curtailment of absence at 182 days has a particularly important effect on the recording of absences caused by tuberculosis.

BDG. 2.-Infective and Parasitic Diseases (Table 3) (International Code Nos. 020-138).

Although the rates are rather erratic, there is a general tendency for single women to have the better experience (Graph 2a and b). The low rates, both for spells and days, in the age-group 35-44, are probably due to the termination of childhood infective diseases. After age 45, the only disease of any numerical importance is herpes zoster. It may be that single women have the better experience because they have less contact with children.

$B D G$. 3.-Neoplasms (Table 4) (International Code Nos. 140-239).

In nearly every age group, single women had the lower incidence of spells. The effect on days of absence was not so marked. There was a definite increase in the average annual duration per person as age increased (Graph 3a and b): this is the same trend as for male conductors given in Health in Industry (1956), but is not so marked at ages over 50. 
10

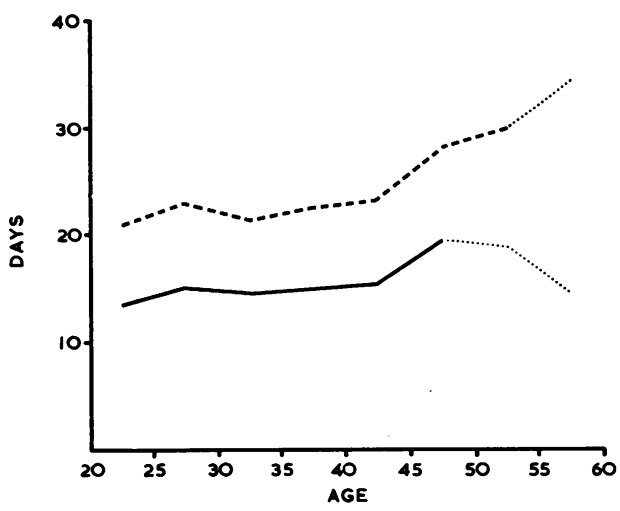

GRAPH 1a.-Average annual duration per person.
ALL DIAGNOSES

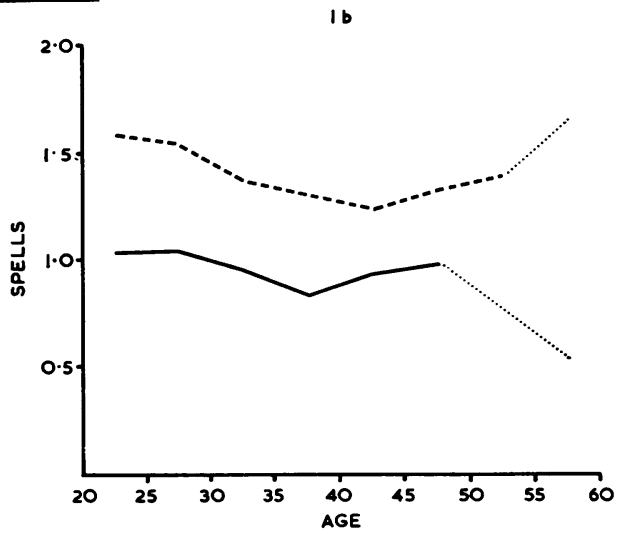

GRAPH 1b.-Annual inception rate (spells).
20

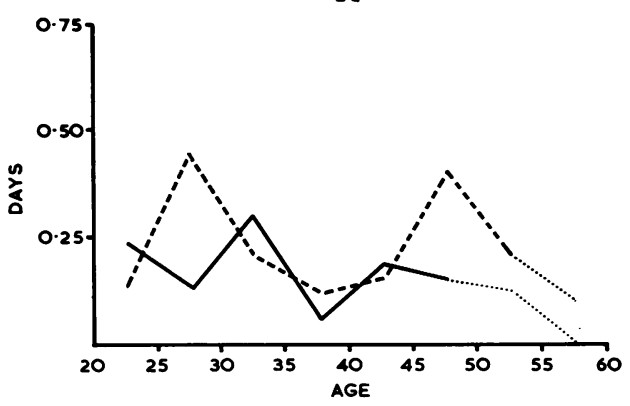

GRAPH 2a.-Average annual duration per person.
B.D.G. 2

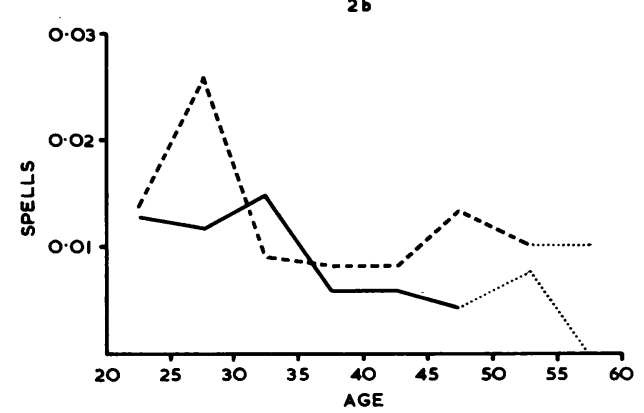

GRAPH 2b.-Annual inception rate (spells).

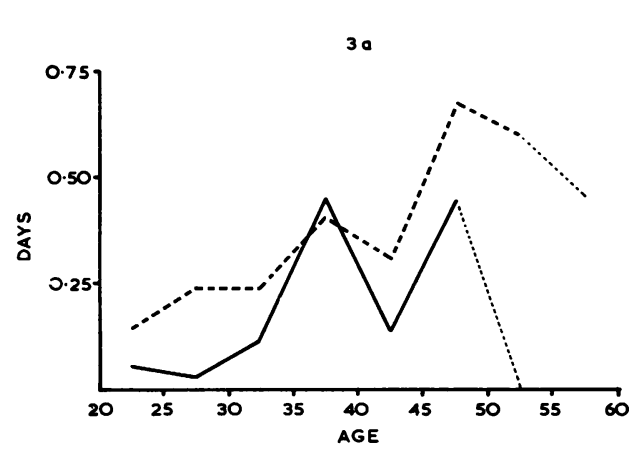

B.D.G. 3

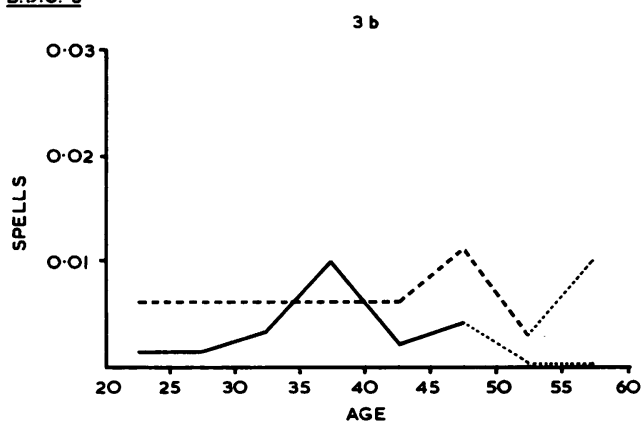

GRAPH 3a.-Average annual duration per person.

GRAPH 3b.-Annual inception rate (spells).

Key: - Single women $(\ldots \ldots$ over age 50$) ; \ldots \ldots$. $\ldots$ - Married women $(\ldots \ldots$ over age 55), 


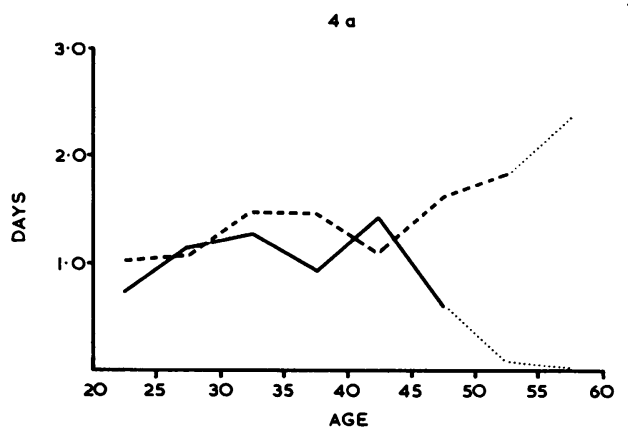

B. D.G. 4

GrAPH 4a.-Average annual duration per person.

Key:

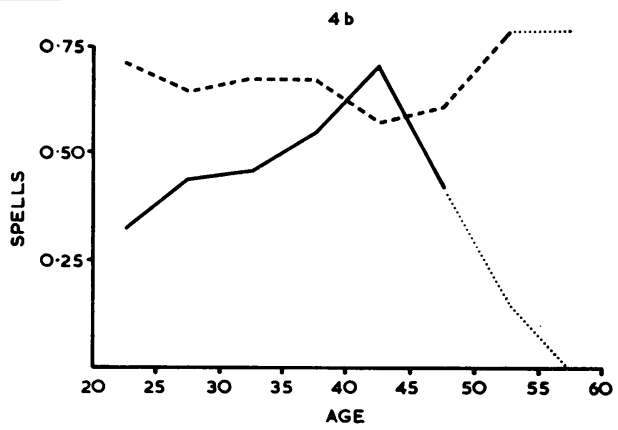

GrAPH 4b.-Annual inception rate (spells).

(50); - . - - Married women (..... over age 55).

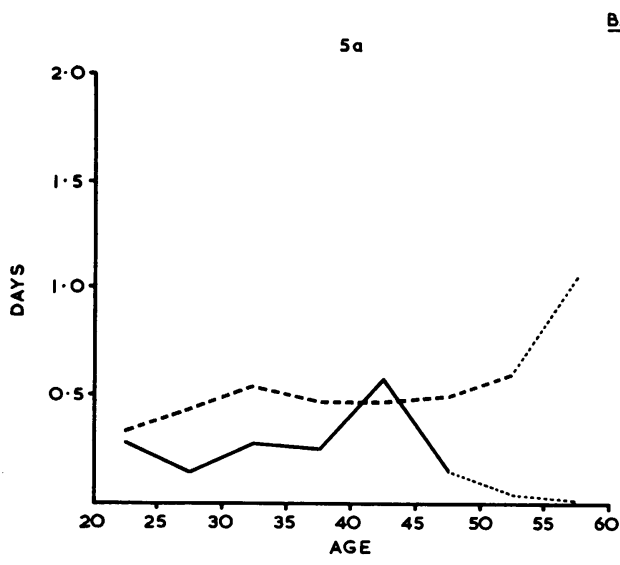

B.D. G. 5

GRAPH 5a.-Average annual duration per person.

Key: - Single women $(\ldots \ldots$ over age 50$)$;

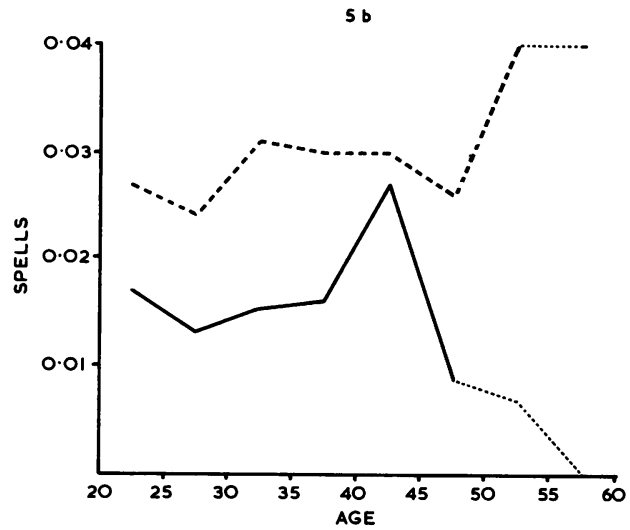

GrAPH 5b.-Annual inception rate (spells).

BDG. 4.-Functional Nervous Disorders (Table 5) (International Code Nos. 300-318).

The annual inception rate (spells) is lower for single women at nearly all ages, but there is little difference between the sickness-absence experience of married and single women as far as the average annual durations per person are concerned. The rise in rates with age at the younger ages of single women is considerable and not unexpected (Graph $4 a$ and $b$ ).

BDG. 5.-Organic Nervous Disorders (Table 6) (International Code Nos. 330-369, 780, 781).

In nearly every age group, the single women's experience is the lower. This is particularly marked after age 45 , when the single women's rates decrease as age increases, but it must be borne in mind that the number of single women bus conductors over age 50 is not very large (Graph $5 a$ and b).
BDG. 6.-Diseases of the Eye (Table 7) (International Code Nos. 370-389).

In this diagnostic group, the rates for married and single women are similar (Graph 6a and b).

BDG. 7.-Diseases of the Ear (Table 8) (International Code Nos. 390-398).

As in diseases of the eye, there is little difference between the rates for married and single women. The diagnoses included in groups 6 and 7 are usually precise, and in neither group is there any reason to expect any differences in incidence between married and single women (Graph 7a and b).

BDG. 8.-Diseases of the Circulatory System (Table 9) International Code Nos. 400-468, 782).

Both rates are consistently lower for single women (Graph 8a and b). There is a considerable increase in the rates for married women after age 45. 

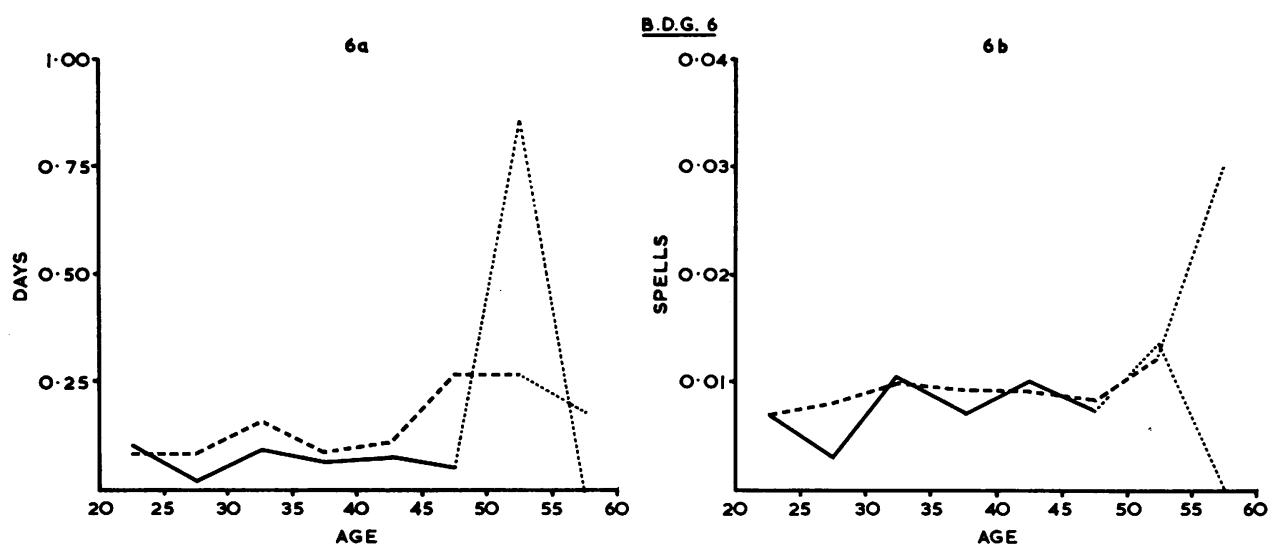

GRAPH 6a.-Average annual duration per person.

GRAPH 6b.-Annual inception rate (spells).

Key

Single women $(. . \ldots$ over age

- Married women (...... over age 55).
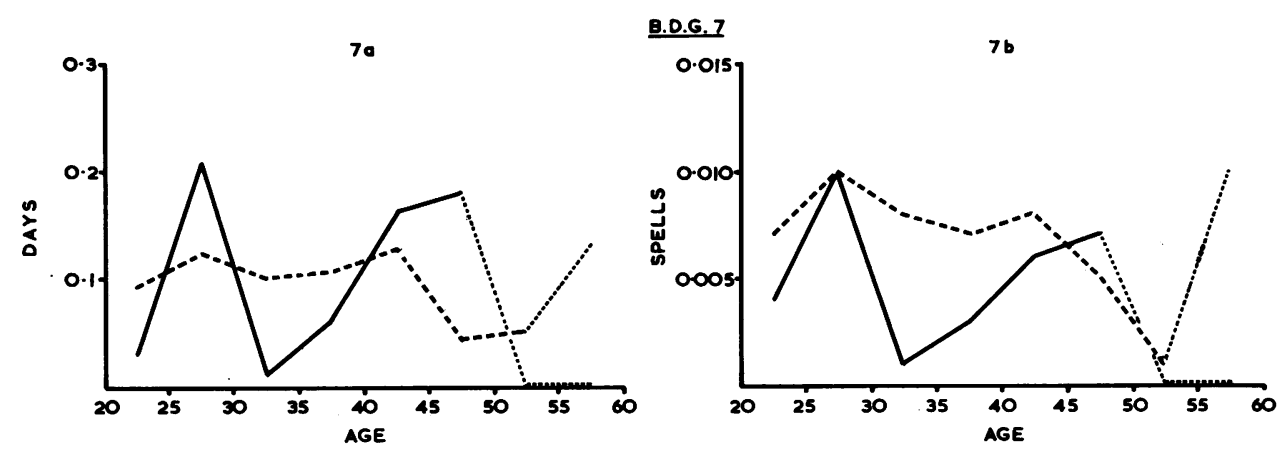

GRAPH 7a.-Average annual duration per person.

GRAPH 7b.-Annual inception rate (spells).

Key: $\longrightarrow$ Single women $(\ldots \ldots$ over age 50$)$; ------ Married women $(\ldots \ldots$ over age 55$)$
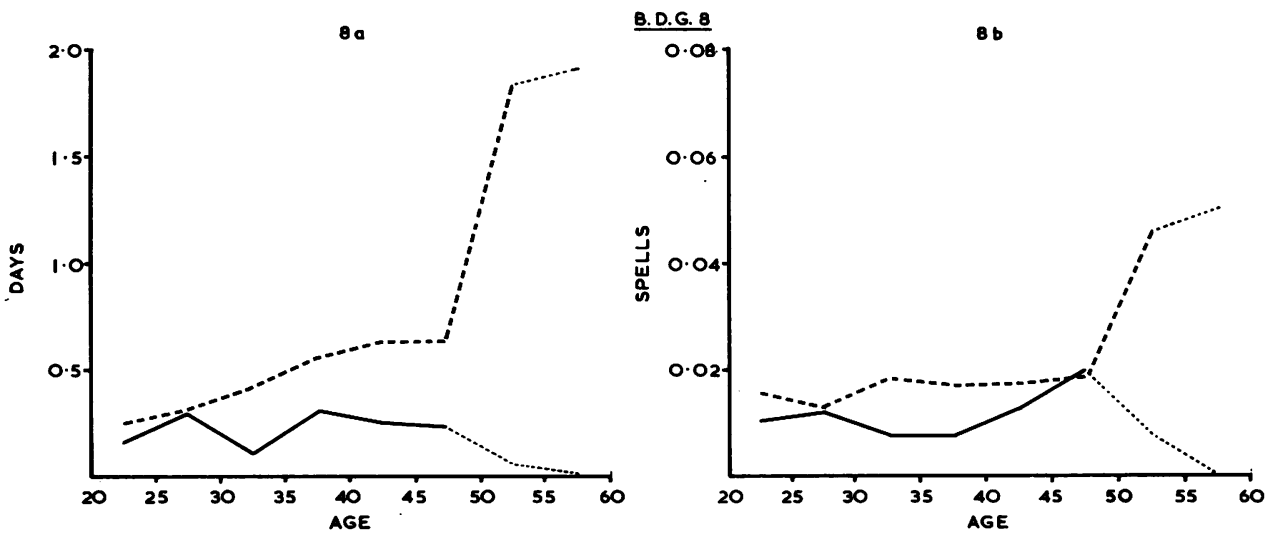

GRAPH 8a.-Average annual duration per person.

GRAPH 8b.-Annual inception rate (spells).

Key:

- Married women (..... over age 55). 

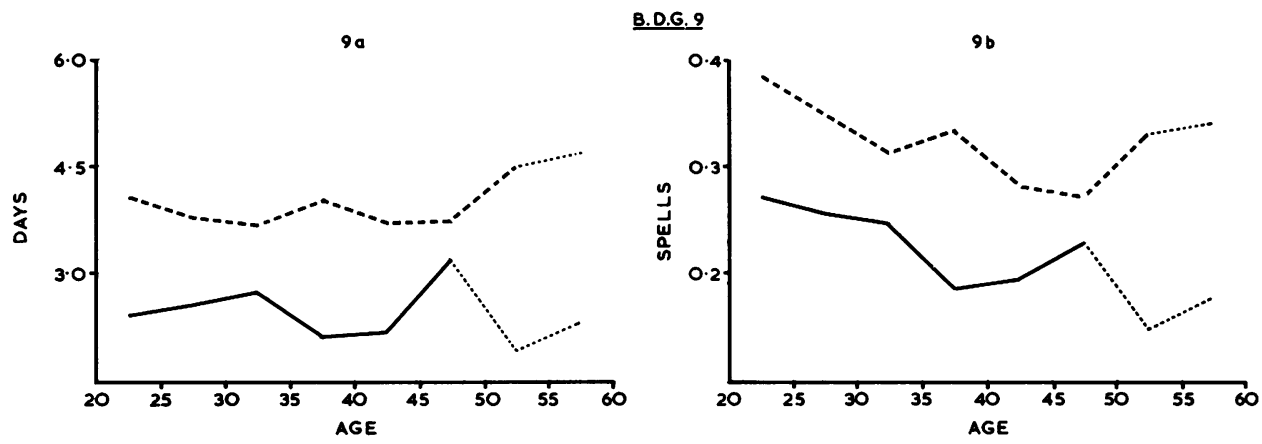

GRAPH 9a.-Average annual duration per person.

Key: $\longrightarrow$ Single women $(\ldots \ldots$ over age 50$) ;------$ Married women $(\ldots \ldots$ over age 55$)$.
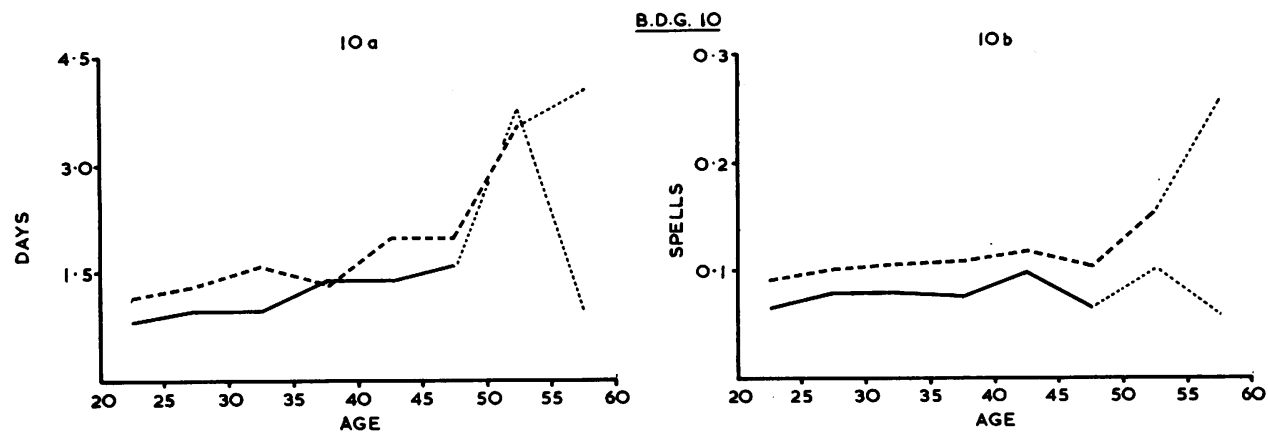

GRAPH 10a.-Average annual duration per person.

Key: $\longrightarrow$ Single women $(\ldots \ldots$ over age 50$) ;-\ldots--$ Married women $(\ldots \ldots$ over age 55).

GRAPH 10b.-Annual inception rate (spells).

Varicose veins are included in this group and are known to be more common among married women.

BDG. 9.-Colds and Influenza (Table 10) (International Code Nos. 470, 480-483).

This group accounts for about one-quarter of the spells of more than three days' duration of single and married women. Rates are, however, considerably lower for single women than for married women, in some age groups by as much as $50 \%$ for spells and over $100 \%$ for days per person (Graph 9a and b). It will be remembered that colds and influenza are thought to be more common in families with school children.

BDG. 10.-Bronchitis (Table 11) (International Code Nos. 500-502).

The annual inception rate (spells) for single women is consistently lower than for married women, but the differences in days of absence are not so large (Graph 10a and b). As might be expected, the average length of spell increases with age. It has not been possible to investigate geographical variations in this paper. Our recent paper on bronchitis (Cornwall and Raffle, 1961) discusses this point. Differences in the smoking habits of single and married women may account for the differences noted, but there is no evidence to support this.

BDG. 11.-Other Respiratory Diseases (Table 12) (International Code Nos. 471-475, 490-493, 510-527, 763, 783).

This is a heterogeneous group, and no firm conclusions are possible. Although the annual inception rate (spells) for single women is consistently lower at ages up to 50, the percentage excess for married women is only about $20 \%$. The pattern is less clear for average annual duration (Graph 11a and b). The incidence of spells decreases as age increases, up to age 50. There was a similar trend for male bus conductors for the previous quinquennium, given in Health in Industry (1956).

BDG. 12.-Diseases of the Stomach and Duodenum (Table 13) (International Code Nos. 540-545, 784).

No clear pattern emerges here (Graph 12a and b). There is no increase in the incidence of this type of 

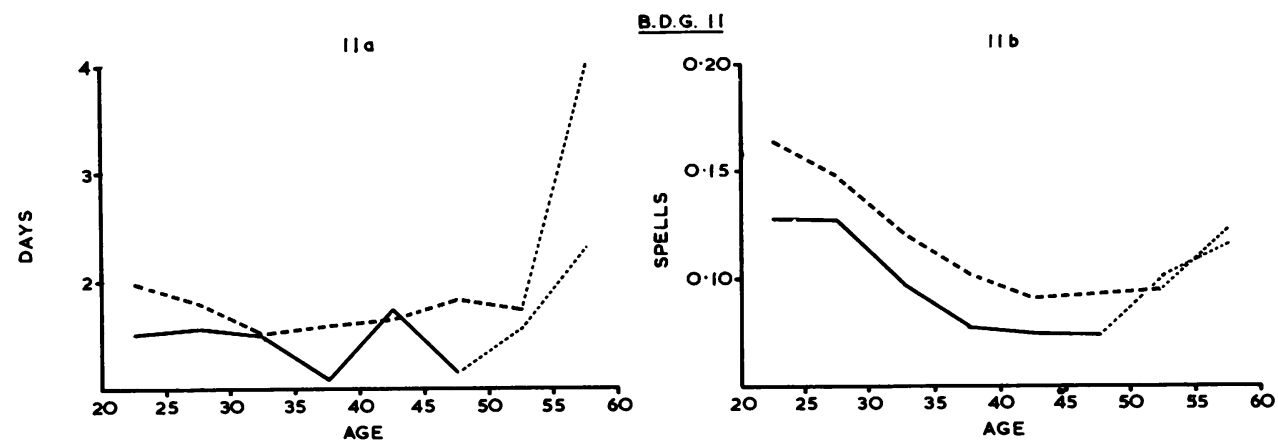

GRAPH 11a.-Average annual duration per person.

GRAPH 11b.-Annual inception rate (spells).

Key: $\longrightarrow$ Single women (..... over age 50); $\ldots \ldots$ over age 55).

disease as age increases. This is in marked contrast to the results for men given in Health in Industry (1956), and referred to in detail by Raffle (1959).

BDG. 13.- Hernia of Abdominal Cavity (Table 14) (International Code Nos. 560-561).
There are very few data in this diagnostic group BDG. 14.-Other Diseases of the Digestive System (Table 15) (International Code Nos. 530-539, 550-553, 570-587, 764, 785).

This is a heterogeneous group, but at all ages,
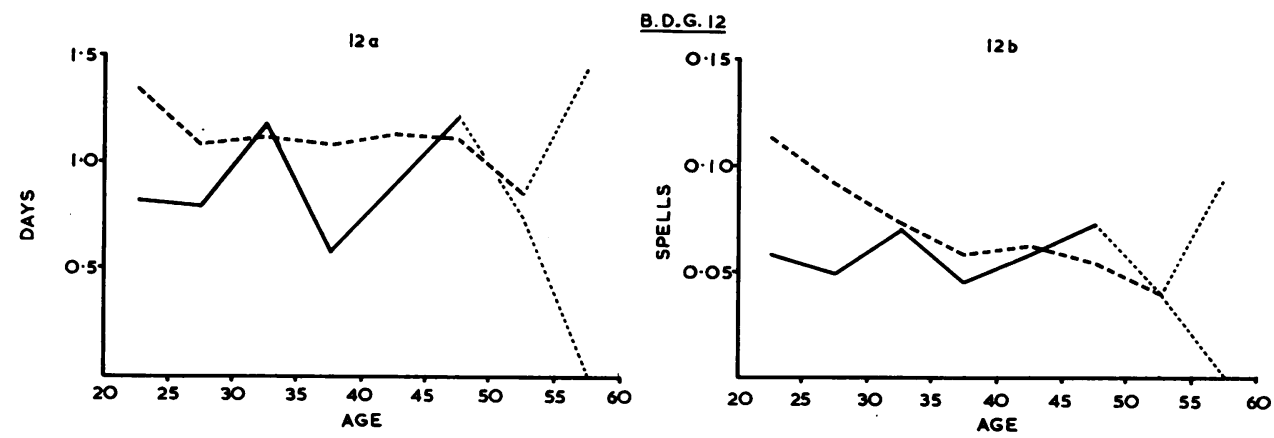

GrAPH 12a.-Average annual duration per person.

Key: $\longrightarrow$ Single women $(\ldots \ldots$ over age 50$)$;

GrAPH 12b.-Annual inception rate (spells). 50); - - - - Married women (..... over age 55).
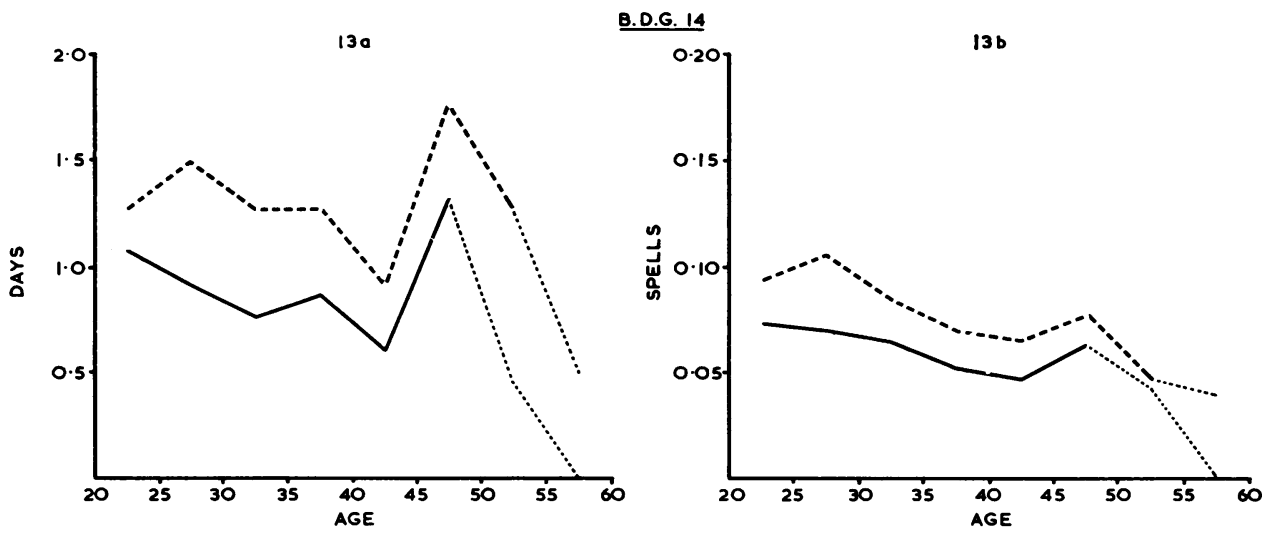

GrAPH 13a.-Average annual duration per person.

GraPH 13b.-Annual inception rate (spells) Key: $\longrightarrow$ Single women $(\ldots \ldots$ over age 50$) ;-----$ Married women (..... over age 55). 

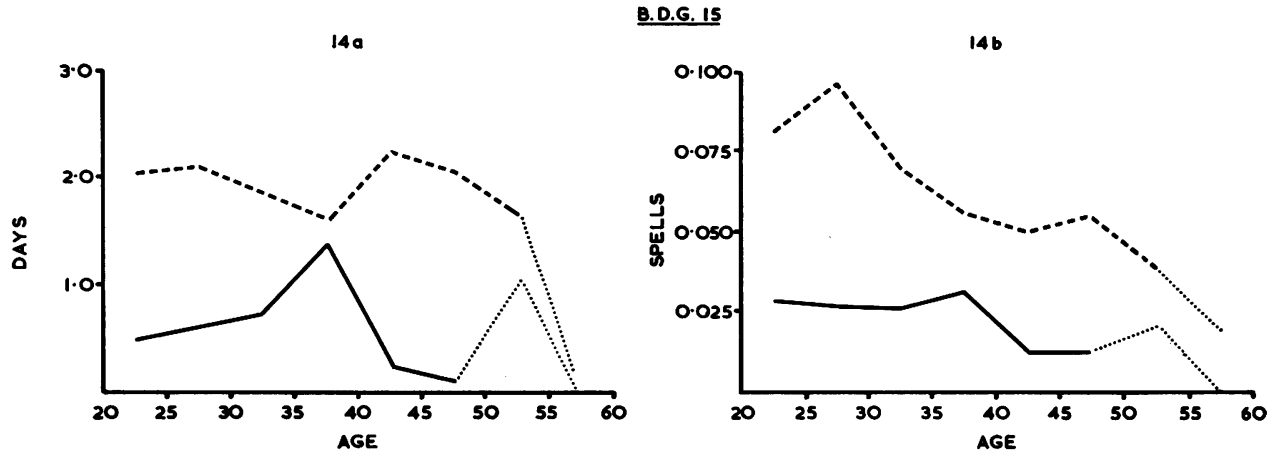

GRAPH 14a.-Average annual duration per person. Key: _ Single women $(\ldots \ldots$ over age 50$)$

GRAPH 14b.-Annual inception rate (spells). - Married women (..... over age 55).

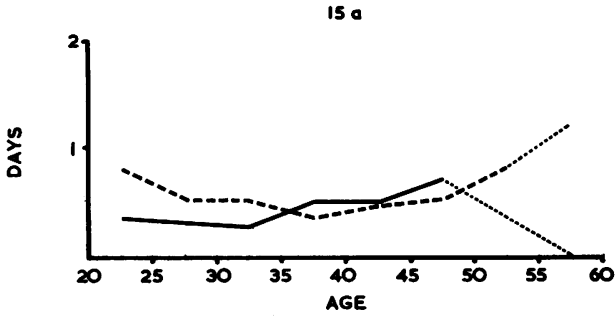

GrAPH 15a.-Average annual duration per person. Key: —_ Single women $(\ldots \ldots$ over age 50$)$

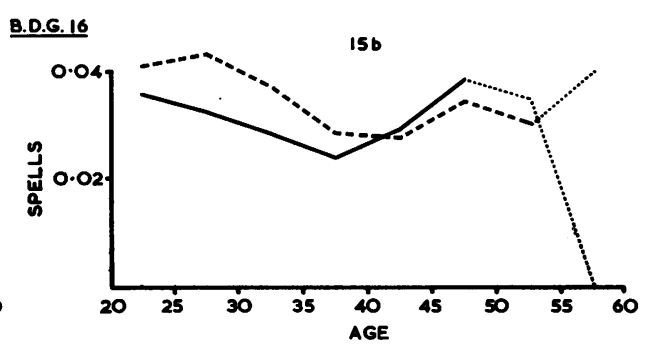

GraPH 15b.-Annual inception rate (spells). married women's rates are higher (Graph 13a and b) than those of single women. The excess is of the order of $30 \%$ for spells and $35 \%$ for days.

In contrast to the experience of male bus conductors, given in Health in Industry (1956), the incidence of spells of sickness absence attributed to this diagnostic group decreases as age increases, both for married and single women conductors.

BDG. 15.-Diseases of Women (Table 16) (International Code Nos. 620-689).

At all ages, single women's rates are lower, by an appreciable margin, as would be expected (Graph $14 \mathrm{a}$ and $\mathrm{b}$ ).

BDG. 16.-Diseases of the Skin (Table 17) (International Code Nos. 690-698, 700-716).

This group shows little difference between the rates of married and single women, and little variation with age (Graph 15a and $b$ ). If the use of modern detergents is a factor in the incidence of sickness absence in this diagnostic group, it might have been expected that married women's rates would have been the higher.

BDG. 17.-Diseases of Bones and Organs of
Movement (Table 18) (International Code Nos. 720-749, 787).

The incidence of sickness absence attributed to this group appears to increase generally with age. For the annual inception rate (spells), single women's rates are consistently lower than those of married women, but for days, the differences are not so marked (Graph 16a and b).

BDG. 18.-Accidents on Duty (Table 19) (International Code Nos. (N) 800-999).

Single women bus conductors experience lower rates of absence caused by accidents on duty. It does not necessarily follow, however, that single women bus conductors have less accidents than married women bus conductors, but merely that they have fewer absences (of more than three days' duration) resulting from accidents (Graph 17a and b).

BDG. 19.-Accidents off Duty (Table 20) (International Code Nos. (N) 800-999).

In this group again, the single women's rates are predominantly lower. This should possibly be expected, as most married women undertake the dual task of conducting a bus and running a home. 


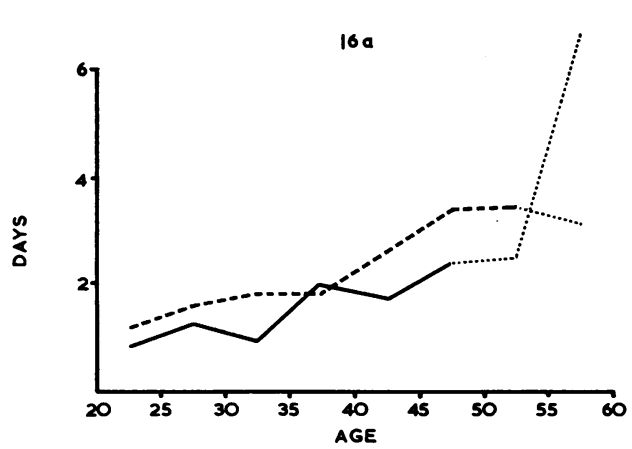

B.D.G.17

GRAPH 16a.-Average annual duration per person.

Key:

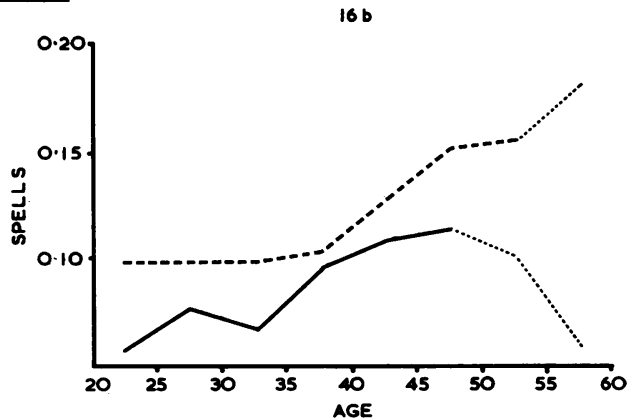

GrAPH 16b.-Annual inception rate (spells).

50); -...- - Married women (..... over age 55).
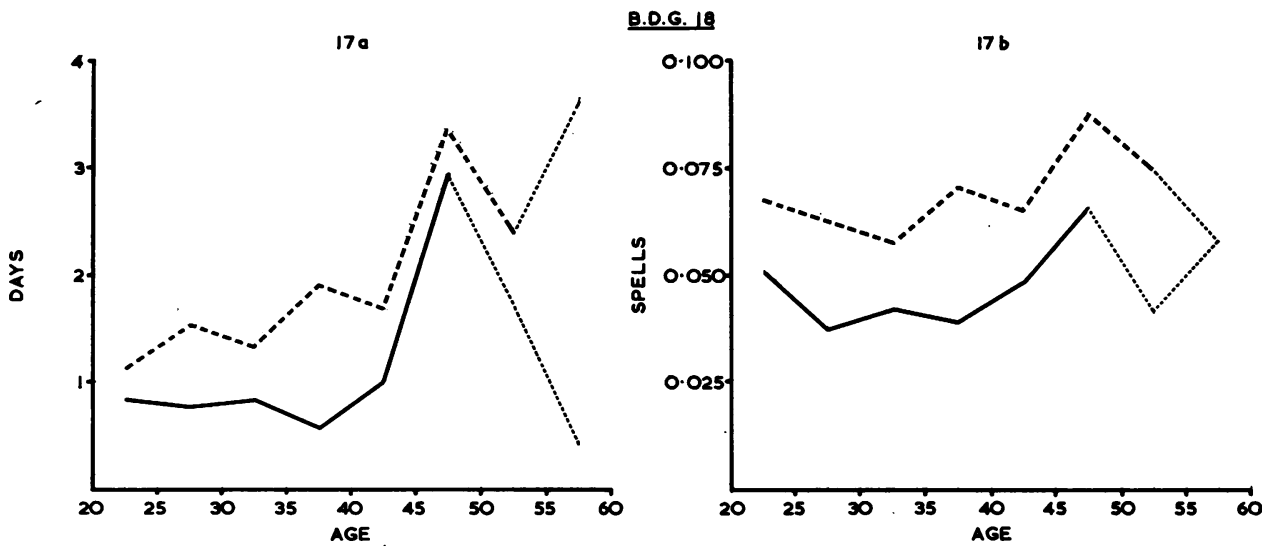

GraPH 17a.-Average annual duration per person.

GRAPH 17b.-Annual inception rate (spells).

Key: _— Single women $(\ldots \ldots$ over age 50$)$;

- Married women (...... over age 55).
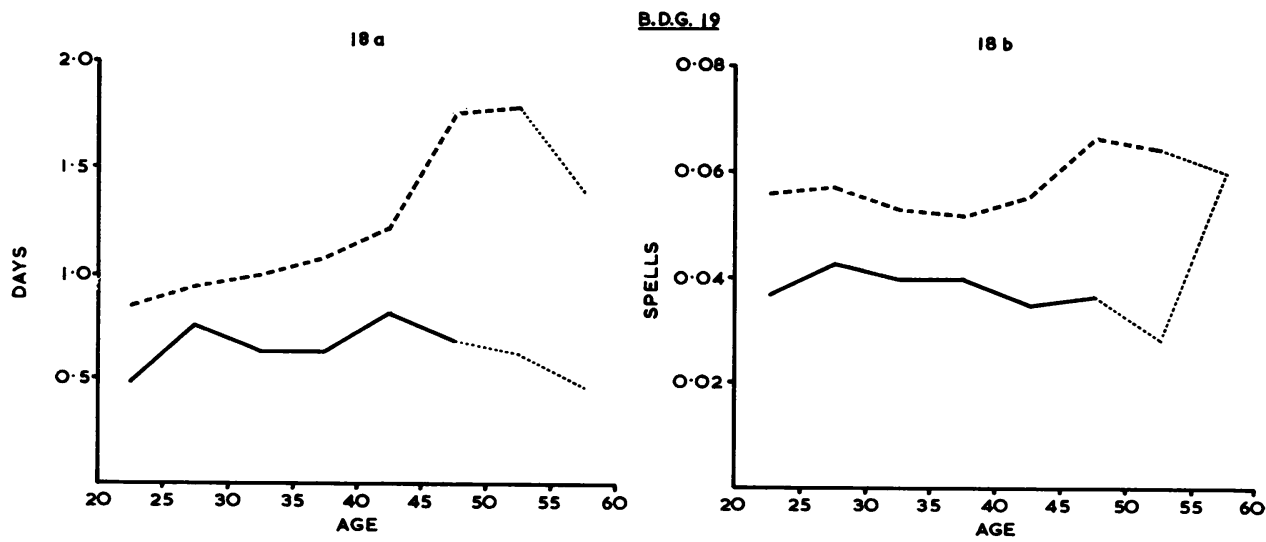

GRAPH 18a.-Average annual duration per person.

GraPH 18b.-Annual inception rate (spells).

Key: - Single women $(\ldots \ldots$ over age 50$)$;

- - Married women (...... over age 55) 

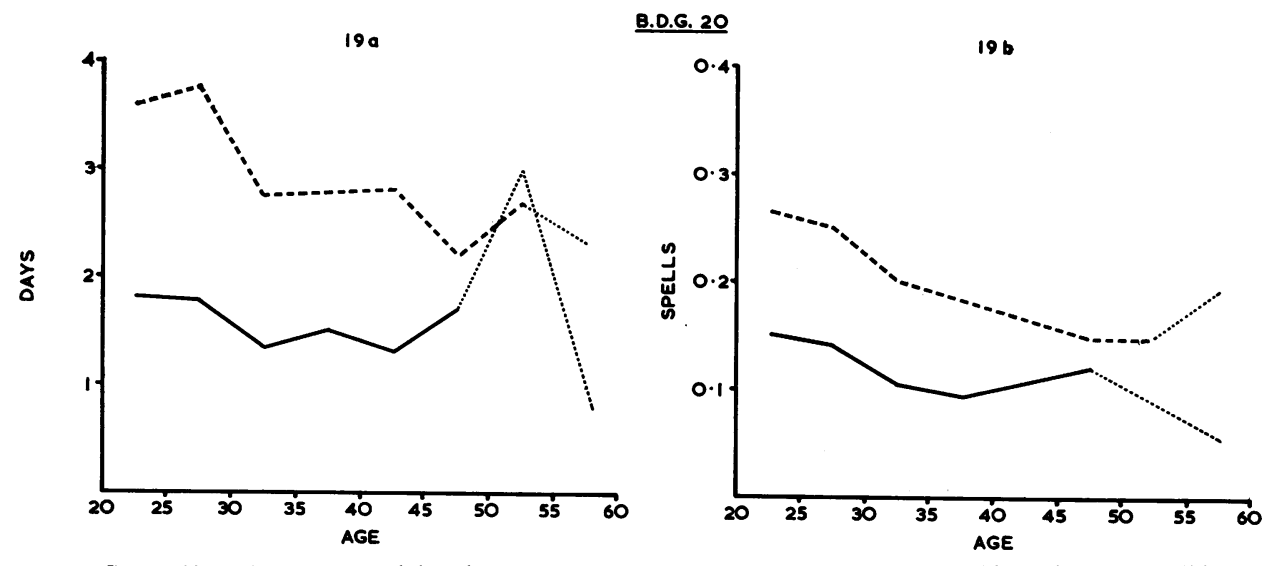

GrAPH 19a.-Average annual duration per person. Key: - Single women $(\ldots \ldots$ over age 50$)$

GRAPH 19b.-Annual inception rate (spells).

There is no general increase of spells with age. (See Graph 18a and b.)

BDG. 20.-Miscellaneous (Table 21) (International Code Nos. 000, 240-299, 320-326, 590-617, 750-762, 765-776, 786, 788-795).

The lower rates for single women are probably only a reflection of the general tendency in most diagnostic groups (Graph 19a and b).

\section{Conclusion}

It may be concluded from the above analysis that single women bus conductors experience appreciably less sickness absence than their married women colleagues. The extent of the difference for each diagnostic group may best be measured by the ratio of the rate (for spells or days) for all married women to the rate for all single women, the rates being indirectly standardized to allow for the different age distributions of the two groups. The following Table sets out the results.

It will be observed that, for most diagnostic groups, the percentage differences are very similar to the average for all diagnoses. This suggests that there is a propensity, of a social origin, for married women to have more absences: it is spread over all diagnoses and the resulting increase in rates is varied only (a) downwards, in the relatively few diagnostic groups where a condition can be accurately diagnosed, or (b) upwards, where there are sound medical reasons for a difference in actual morbidity.

We wish to express our thanks to Mr. F. H. Spratling, F.I.A., Chief Establishment Officer, and Dr. L. G.

TABLE

PERCENTAGE RATIO OF SICKNESS OF MARRIED WOMEN TO THAT OF SINGLE WOMEN, ADJUSTED FOR AGE

\begin{tabular}{|c|c|c|c|}
\hline $\begin{array}{c}\text { Broad Diagnostic } \\
\text { Group } \\
\text { No. }\end{array}$ & Short Descriptions & $\begin{array}{l}\text { \% Ratio of Average } \\
\text { Annual Durations } \\
\text { (Days) }\end{array}$ & $\begin{array}{l}\text { \% Ratio of Annual } \\
\text { Inception Rates } \\
\text { (Spells) }\end{array}$ \\
\hline \multirow[t]{2}{*}{$\begin{array}{r}1 \\
2 \\
3 \\
4 \\
5 \\
6 \\
7 \\
8 \\
9 \\
10 \\
11 \\
12 \\
13 \\
14 \\
15 \\
16 \\
17 \\
18 \\
19 \\
20\end{array}$} & $\begin{array}{l}\text { Tuberculosis } \\
\text { Infective and parasitic diseases } \\
\text { Neoplasms } \\
\text { Functional nervous disorders } \\
\text { Organic nervous disorders } \\
\text { Diseases of the eye } \\
\text { Diseases of the ear } \\
\text { Diseases of the circulatory system } \\
\text { Colds and influenza } \\
\text { Bronchitis } \\
\text { Other respiratory diseases } \\
\text { Diseases of the stomach and duodenum } \\
\text { Hernia of abdominal cavity } \\
\text { Other diseases of the digestive system } \\
\text { Diseases of women } \\
\text { Diseases of the skin } \\
\text { Diseases of bones and organs of movement } \\
\text { Accidents on duty } \\
\text { Accidents off duty } \\
\text { Miscellaneous }\end{array}$ & $\begin{array}{l}159 \\
134 \\
195 \\
124 \\
161 \\
134 \\
100 \\
197 \\
144 \\
119 \\
117 \\
122 \\
367 \\
140 \\
280 \\
129 \\
130 \\
155 \\
155 \\
175\end{array}$ & $\begin{array}{r}117 \\
132 \\
200 \\
138 \\
166 \\
117 \\
143 \\
149 \\
135 \\
133 \\
120 \\
130 \\
1,205 \\
133 \\
267 \\
113 \\
133 \\
139 \\
138 \\
164\end{array}$ \\
\hline & All diagnostic groups & 147 & 140 \\
\hline
\end{tabular}


Norman, Chief Medical Officer, of London Transport, for their help and encouragement in the preparation of this paper, to Mr. J. E. Ager and the staff of the Central Record and Actuarial Sections for the machine work and computation involved, and to Mrs. A. Garey who has drawn the graphs.

\section{REFERENCES}

Cornwall, C. J., and Raffle, P. A. B. (1961). Brit. J. industr. Med., 18,

London Transport Executive (1956). Health in Industry: A Contribution to the Study of Sickness Absence. Butterworth, London.

Raffle, P. A. B. (1959). Lancet, 2. 839.

\section{A P P E N D I X}

\section{ABSENCES OF FOUR DAYS DURATION OR MORE IN MARRIED AND SINGLE WOMEN BUS CONDUCTORS, 1953-1957.}

TABLE 1: ALL DIAGNOSES

\begin{tabular}{|c|c|c|c|c|c|c|c|c|c|c|c|c|}
\hline \multirow[t]{2}{*}{ Age Group } & \multicolumn{2}{|c|}{$\begin{array}{l}\text { Exposure to Risk } \\
\text { (Man Years) }\end{array}$} & \multicolumn{2}{|c|}{$\begin{array}{l}\text { No. of Days } \\
\text { Sickness Absence* }\end{array}$} & \multicolumn{2}{|c|}{$\begin{array}{c}\text { Average Annual } \\
\text { Duration Per Person } \\
\text { (Days) }\end{array}$} & \multicolumn{2}{|c|}{ No. of Spells } & \multicolumn{2}{|c|}{$\begin{array}{c}\text { Annual Inception } \\
\text { Rate } \\
\text { (Spells) }\end{array}$} & \multicolumn{2}{|c|}{$\begin{array}{l}\text { Average Length } \\
\text { of Spell } \\
\text { (Days) }\end{array}$} \\
\hline & Single & Married & Single & Married & Single & Married & Single & Married & Single & Married & Single & Married \\
\hline $\begin{array}{l}\text { Up to } 24 \\
25-29 \\
30-34 \\
35-39 \\
40-44 \\
45-49 \\
50-54 \\
55-59 \\
\text { (60 and } \\
\text { over) }\end{array}$ & $\begin{array}{r}2,100 \\
1,668 \\
1,082 \\
694 \\
625 \\
460 \\
142 \\
17 \\
-\end{array}$ & $\begin{array}{r}904 \\
1,971 \\
2,447 \\
2,611 \\
2,679 \\
1,898 \\
670 \\
100 \\
\\
15\end{array}$ & $\begin{array}{r}27,413 \\
24,513 \\
15,434 \\
10,160 \\
9,637 \\
8,889 \\
2,642 \\
239 \\
-\end{array}$ & $\begin{array}{r}19,467 \\
45,835 \\
52,561 \\
58,623 \\
62,358 \\
53,037 \\
20,174 \\
3,394 \\
665\end{array}$ & $\begin{array}{r}13.049 \\
14.683 \\
14.261 \\
14.630 \\
15.419 \\
19.325 \\
18.671 \\
14.059 \\
-\end{array}$ & $\begin{array}{l}21 \cdot 550 \\
23 \cdot 238 \\
21 \cdot 497 \\
22 \cdot 453 \\
23 \cdot 260 \\
27 \cdot 950 \\
30 \cdot 079 \\
34 \cdot 110 \\
44 \cdot 334\end{array}$ & $\begin{array}{r}2,151 \\
1,710 \\
1,026 \\
599 \\
587 \\
446 \\
111 \\
10 \\
-\end{array}$ & $\begin{array}{r}1,442 \\
3,057 \\
3,355 \\
3,449 \\
3,318 \\
2,468 \\
920 \\
162 \\
19\end{array}$ & $\begin{array}{r}1.024 \\
1.024 \\
0.948 \\
0.863 \\
0.939 \\
0.970 \\
0.784 \\
0.588 \\
-\end{array}$ & $\begin{array}{l}1 \cdot 596 \\
1.550 \\
1 \cdot 372 \\
1 \cdot 321 \\
1 \cdot 238 \\
1.301 \\
1 \cdot 372 \\
1.628 \\
1 \cdot 267\end{array}$ & $\begin{array}{l}12 \cdot 7 \\
14 \cdot 3 \\
15 \cdot 0 \\
17 \cdot 0 \\
16 \cdot 4 \\
19 \cdot 9 \\
23 \cdot 8 \\
23 \cdot 9 \\
-\end{array}$ & $\begin{array}{l}13.5 \\
15.0 \\
15.7 \\
17 \cdot 0 \\
18.8 \\
21.5 \\
21.9 \\
21.0 \\
35.0\end{array}$ \\
\hline Total & 6,788 & 13,295 & 98,927 & 316,114 & - & - & 6,640 & 18,190 & - & - & - & - \\
\hline
\end{tabular}

TABLE 2: BROAD DIAGNOSTIC GROUP I: TUBERCULOSIS

(I.S.C. Nos. 001-019)

\begin{tabular}{|c|c|c|c|c|c|c|c|c|c|c|c|c|}
\hline \multirow[t]{2}{*}{ Age Group } & \multicolumn{2}{|c|}{$\begin{array}{l}\text { Exposure to Risk } \\
\text { (Man Years) }\end{array}$} & \multicolumn{2}{|c|}{$\begin{array}{c}\text { No. of Days } \\
\text { Sickness Absence* }\end{array}$} & \multicolumn{2}{|c|}{$\begin{array}{c}\text { Average Annual } \\
\text { Duration Per Person } \\
\text { (Days) }\end{array}$} & \multicolumn{2}{|c|}{ No. of Spells } & \multicolumn{2}{|c|}{$\begin{array}{c}\text { Annual Inception } \\
\text { Rate } \\
\text { (Spells) }\end{array}$} & \multicolumn{2}{|c|}{$\begin{array}{l}\text { Average Length } \\
\text { of Spell } \\
\text { (Days) }\end{array}$} \\
\hline & Single & Married & Single & Married & Single & Married & Single & Married & Single & Married & Single & Married \\
\hline $\begin{array}{l}\text { Up to } 24 \\
25-29 \\
30-34 \\
35-39 \\
40-44 \\
45-49 \\
50-54 \\
55-59 \\
\text { (60 and } \\
\text { over) }\end{array}$ & $\begin{array}{r}2,100 \\
1,668 \\
1,082 \\
694 \\
625 \\
460 \\
142 \\
17 \\
-\end{array}$ & $\begin{array}{r}904 \\
1,971 \\
2,447 \\
2,611 \\
2,679 \\
1,898 \\
670 \\
100 \\
15\end{array}$ & $\begin{array}{l}260 \\
574 \\
180 \\
= \\
= \\
= \\
-\end{array}$ & $\begin{array}{r}179 \\
1,113 \\
392 \\
326 \\
182 \\
=\end{array}$ & $\begin{array}{c}0 \cdot 124 \\
0 \cdot 344 \\
0 \cdot 166 \\
= \\
= \\
= \\
-\end{array}$ & $\begin{array}{c}0.198 \\
0.564 \\
0.160 \\
0.125 \\
-\overline{-} \\
0.096 \\
=\end{array}$ & $\begin{array}{l}3 \\
4 \\
- \\
- \\
= \\
-\end{array}$ & $\begin{array}{l}-5 \\
2 \\
3 \\
1 \\
-\end{array}$ & $\begin{array}{c}0.001 \\
0.002 \\
= \\
= \\
= \\
= \\
-\end{array}$ & $\begin{array}{c}0 . \overline{003} \\
0.001 \\
0.001 \\
\overline{0} \\
= \\
- \\
-\end{array}$ & $\begin{array}{c}86 \cdot 7 \\
143 \cdot 5 \\
= \\
= \\
= \\
-\end{array}$ & $\begin{array}{c}2 \overline{222.6} \\
196.0 \\
108 \cdot 7 \\
- \\
182.0 \\
- \\
-\end{array}$ \\
\hline Total & 6,788 & 13,295 & 1,014 & 2,192 & - & - & 7 & 11 & - & 一 & - & - \\
\hline
\end{tabular}

TABLE 3: BROAD DIAGNOSTIC GROUP 2: INFECTIVE AND PARASITIC DISEASES (I.S.C. Nos. 020-138)

\begin{tabular}{|c|c|c|c|c|c|c|c|c|c|c|c|c|}
\hline \multirow[t]{2}{*}{ Age Group } & \multicolumn{2}{|c|}{$\begin{array}{l}\text { Exposure to Risk } \\
\text { (Man Years) }\end{array}$} & \multicolumn{2}{|c|}{$\begin{array}{c}\text { No. of Days } \\
\text { Sickness Absence* }\end{array}$} & \multicolumn{2}{|c|}{$\begin{array}{c}\text { Average Annual } \\
\text { Duration Per Person } \\
\text { (Days) }\end{array}$} & \multicolumn{2}{|c|}{ No. of Spells } & \multicolumn{2}{|c|}{$\begin{array}{c}\text { Annual Inception } \\
\text { Rate } \\
\text { (Spells) }\end{array}$} & \multicolumn{2}{|c|}{$\begin{array}{l}\text { Average Length } \\
\text { of Spell } \\
\text { (Days) }\end{array}$} \\
\hline & Single & Married & Single & Married & Single & Married & Single & Married & Single & Married & Single & Married \\
\hline $\begin{array}{l}\text { Up to } 24 \\
25-29 \\
30-34 \\
35-39 \\
40-44 \\
45-49 \\
50-54 \\
55-59 \\
\text { (60 and } \\
\text { over) }\end{array}$ & $\begin{array}{r}2,100 \\
1,668 \\
1,082 \\
694 \\
625 \\
460 \\
142 \\
17 \\
-\end{array}$ & $\begin{array}{r}904 \\
1,971 \\
2,447 \\
2,611 \\
2,679 \\
1,898 \\
670 \\
100 \\
15\end{array}$ & $\begin{array}{r}500 \\
203 \\
322 \\
43 \\
102 \\
68 \\
16 \\
- \\
-\end{array}$ & $\begin{array}{r}113 \\
891 \\
499 \\
278 \\
390 \\
752 \\
137 \\
9 \\
-\end{array}$ & $\begin{array}{r}0 \cdot 238 \\
0 \cdot 122 \\
0 \cdot 298 \\
0 \cdot 062 \\
0 \cdot 163 \\
0 \cdot 148 \\
0 \cdot 113 \\
- \\
-\end{array}$ & $\begin{array}{r}0.125 \\
0.452 \\
0.204 \\
0.106 \\
0.145 \\
0.396 \\
0.204 \\
0.090 \\
-\end{array}$ & $\begin{array}{r}28 \\
20 \\
16 \\
4 \\
4 \\
2 \\
1 \\
- \\
-\end{array}$ & $\begin{array}{r}13 \\
51 \\
22 \\
21 \\
22 \\
26 \\
7 \\
1 \\
-\end{array}$ & $\begin{array}{r}0.013 \\
0.012 \\
0.015 \\
0.006 \\
0.006 \\
0.004 \\
0.007 \\
- \\
-\end{array}$ & $\begin{array}{r}0.014 \\
0.026 \\
0.009 \\
0.008 \\
0.008 \\
0.014 \\
0.010 \\
0.010 \\
-\end{array}$ & $\begin{array}{r}17 \cdot 9 \\
10 \cdot 2 \\
20 \cdot 1 \\
10 \cdot 8 \\
25 \cdot 5 \\
34 \cdot 0 \\
16 \cdot 0 \\
- \\
-\end{array}$ & $\begin{array}{r}8 \cdot 7 \\
17.5 \\
22.7 \\
13.2 \\
17.7 \\
28.9 \\
19.6 \\
9.0 \\
-\end{array}$ \\
\hline Total & 6,788 & 13,295 & 1,254 & 3,069 & - & 一 & 75 & 163 & 一 & - & - & - \\
\hline
\end{tabular}

*Excludes days beyond first six months of absence. 
TABLE 4: BROAD DIAGNOSTIC GROUP 3: NEOPLASMS (I.S.C. Nos. 140-239)

\begin{tabular}{|c|c|c|c|c|c|c|c|c|c|c|c|c|}
\hline \multirow[t]{2}{*}{ Age Group } & \multicolumn{2}{|c|}{$\begin{array}{l}\text { Exposure to Risk } \\
\text { (Man Years) }\end{array}$} & \multicolumn{2}{|c|}{$\begin{array}{c}\text { No. of Days } \\
\text { Sickness Absence* }\end{array}$} & \multicolumn{2}{|c|}{$\begin{array}{c}\text { Average Annual } \\
\text { Duration Per Person } \\
\text { (Days) }\end{array}$} & \multicolumn{2}{|c|}{ No. of Spells } & \multicolumn{2}{|c|}{$\begin{array}{c}\text { Annual Inception } \\
\text { Rate } \\
\text { (Spells) }\end{array}$} & \multicolumn{2}{|c|}{$\begin{array}{l}\text { Average Length } \\
\text { of Spell } \\
\text { (Days) }\end{array}$} \\
\hline & Single & Married & Single & Married & Single & Married & Single & Married & Single & Married & Single & Married \\
\hline $\begin{array}{l}\text { Up to } 24 \\
25-29 \\
30-34 \\
35-39 \\
40-44 \\
45-49 \\
50-54 \\
55-59 \\
\text { (60 and } \\
\text { over) }\end{array}$ & $\begin{array}{r}2,100 \\
1,668 \\
1,082 \\
694 \\
625 \\
460 \\
142 \\
17 \\
-\end{array}$ & $\begin{array}{r}904 \\
1,971 \\
2,447 \\
2,611 \\
2,679 \\
1,898 \\
670 \\
100 \\
15\end{array}$ & $\begin{array}{r}103 \\
29 \\
110 \\
309 \\
82 \\
200 \\
- \\
- \\
-\end{array}$ & $\begin{array}{r}134 \\
462 \\
558 \\
1,098 \\
831 \\
1,265 \\
396 \\
44 \\
47\end{array}$ & $\begin{array}{r}0.049 \\
0.017 \\
0.102 \\
0.445 \\
0.131 \\
0.435 \\
- \\
- \\
-\end{array}$ & $\begin{array}{l}0 \cdot 148 \\
0 \cdot 234 \\
0 \cdot 228 \\
0 \cdot 421 \\
0 \cdot 310 \\
0 \cdot 667 \\
0 \cdot 590 \\
0 \cdot 442 \\
3 \cdot 133\end{array}$ & $\begin{array}{r}3 \\
1 \\
3 \\
7 \\
1 \\
2 \\
- \\
- \\
-\end{array}$ & $\begin{array}{r}5 \\
11 \\
14 \\
15 \\
16 \\
21 \\
2 \\
1 \\
-\end{array}$ & $\begin{array}{r}0.001 \\
0.001 \\
0.003 \\
0.010 \\
0.002 \\
0.004 \\
- \\
- \\
-\end{array}$ & $\begin{array}{r}0.006 \\
0.006 \\
0.006 \\
0.006 \\
0.006 \\
0.011 \\
0.003 \\
0.010 \\
-\end{array}$ & $\begin{array}{r}34 \cdot 3 \\
29 \cdot 0 \\
36 \cdot 7 \\
44 \cdot 1 \\
82 \cdot 0 \\
100 \cdot 0 \\
- \\
- \\
-\end{array}$ & $\begin{array}{r}26 \cdot 8 \\
42 \cdot 0 \\
39 \cdot 9 \\
73 \cdot 2 \\
51 \cdot 9 \\
60 \cdot 2 \\
198 \cdot 0 \\
44 \cdot 0 \\
-\end{array}$ \\
\hline Total & 6,788 & 13,295 & 833 & 4,835 & - & - & 17 & 85 & - & - & - & - \\
\hline
\end{tabular}

TABLE 5: BROAD DIAGNOSTIC GROUP 4: FUNCTIONAL NERVOUS DISORDERS (I.S.C. Nos. 300-318)

\begin{tabular}{|c|c|c|c|c|c|c|c|c|c|c|c|c|}
\hline \multirow[t]{2}{*}{ Age Group } & \multicolumn{2}{|c|}{$\begin{array}{c}\text { Exposure to Risk } \\
\text { (Man Years) }\end{array}$} & \multicolumn{2}{|c|}{$\begin{array}{l}\text { No. of Days } \\
\text { Sickness Absence* }\end{array}$} & \multicolumn{2}{|c|}{$\begin{array}{c}\text { Average Annual } \\
\text { Duration Per Person } \\
\text { (Days) }\end{array}$} & \multicolumn{2}{|c|}{ No. of Spells } & \multicolumn{2}{|c|}{$\begin{array}{c}\text { Annual Inception } \\
\text { Rate } \\
\text { (Spells) }\end{array}$} & \multicolumn{2}{|c|}{$\begin{array}{l}\text { Average Length } \\
\text { of Spell } \\
\text { (Days) }\end{array}$} \\
\hline & Single & Married & Single & Married & Single & Married & Single & Married & Single & Married & Single & Married \\
\hline $\begin{array}{l}\text { Up to } 24 \\
25-29 \\
30-34 \\
35-39 \\
40-44 \\
45-49 \\
50-54 \\
55-59 \\
\text { (60 and } \\
\text { over) }\end{array}$ & $\begin{array}{r}2,100 \\
1,668 \\
1,082 \\
694 \\
625 \\
460 \\
142 \\
17 \\
-\end{array}$ & $\begin{array}{r}904 \\
1,971 \\
2,447 \\
2,611 \\
2,679 \\
1,898 \\
670 \\
100 \\
15\end{array}$ & $\begin{array}{r}1,312 \\
1,957 \\
1,433 \\
620 \\
878 \\
287 \\
16 \\
- \\
-\end{array}$ & $\begin{array}{r}945 \\
2,167 \\
3,608 \\
3,975 \\
2,938 \\
2,982 \\
1,193 \\
235 \\
53\end{array}$ & $\begin{array}{r}0.625 \\
1.172 \\
1.324 \\
0.893 \\
1.405 \\
0.624 \\
0.113 \\
=\end{array}$ & $\begin{array}{l}1.046 \\
1.099 \\
1.476 \\
1.522 \\
1.096 \\
1.572 \\
1.779 \\
2.362 \\
3.533\end{array}$ & $\begin{array}{r}70 \\
71 \\
49 \\
39 \\
43 \\
18 \\
2 \\
- \\
-\end{array}$ & $\begin{array}{r}63 \\
127 \\
164 \\
176 \\
154 \\
113 \\
53 \\
8 \\
1\end{array}$ & $\begin{array}{r}0.033 \\
0.043 \\
0.045 \\
0.056 \\
0.069 \\
0.039 \\
0.014 \\
- \\
-\end{array}$ & $\begin{array}{l}0.070 \\
0.064 \\
0.067 \\
0.067 \\
0.057 \\
0.060 \\
0.079 \\
0.080 \\
0.067\end{array}$ & $\begin{array}{r}18 \cdot 7 \\
27 \cdot 6 \\
29 \cdot 2 \\
15 \cdot 9 \\
20 \cdot 4 \\
15 \cdot 9 \\
8 \cdot 0 \\
- \\
-\end{array}$ & $\begin{array}{l}15 \cdot 0 \\
17 \cdot 1 \\
22.0 \\
22.6 \\
19 \cdot 1 \\
26.4 \\
22.5 \\
29.4 \\
53.0\end{array}$ \\
\hline Total & 6,788 & 13,295 & 6,503 & 18,096 & - & - & 292 & 859 & - & - & - & - \\
\hline
\end{tabular}

TABLE 6: BROAD DIAGNOSTIC GROUYP 5: ORGANIC NERVOUS DISORDERS (I.S.C. Nos. 330-369, 780, 781)

\begin{tabular}{|c|c|c|c|c|c|c|c|c|c|c|c|c|}
\hline \multirow[t]{2}{*}{ Age Group } & \multicolumn{2}{|c|}{$\begin{array}{l}\text { Exposure to Risk } \\
\text { (Man Years) }\end{array}$} & \multicolumn{2}{|c|}{$\begin{array}{l}\text { No. of Days } \\
\text { Sickness Absence* }\end{array}$} & \multicolumn{2}{|c|}{$\begin{array}{c}\text { Average Annual } \\
\text { Duration Per Person } \\
\text { (Days) }\end{array}$} & \multicolumn{2}{|c|}{ No. of Spells } & \multicolumn{2}{|c|}{$\begin{array}{c}\text { Annual Inception } \\
\text { Rate } \\
\text { (Spells) }\end{array}$} & \multicolumn{2}{|c|}{$\begin{array}{l}\text { Average Length } \\
\text { of Spell } \\
\text { (Days) }\end{array}$} \\
\hline & Single & Married & Single & Married & Single & Married & Single & Married & Single & Married & Single & Married \\
\hline $\begin{array}{l}\text { Up to } 24 \\
25-29 \\
30-34 \\
35-39 \\
40-44 \\
45-49 \\
50-54 \\
55-59 \\
600 \text { and }\end{array}$ & $\begin{array}{r}2,100 \\
1,668 \\
1,082 \\
694 \\
625 \\
460 \\
142 \\
17\end{array}$ & $\begin{array}{r}904 \\
1,971 \\
2,447 \\
2,611 \\
2,679 \\
1,898 \\
670 \\
100\end{array}$ & $\begin{array}{r}613 \\
237 \\
297 \\
169 \\
359 \\
62 \\
6 \\
-\end{array}$ & $\begin{array}{r}297 \\
814 \\
1,335 \\
1,207 \\
1,216 \\
892 \\
389 \\
136\end{array}$ & $\begin{array}{l}0.292 \\
0.142 \\
0.274 \\
0.243 \\
0.574 \\
0.135 \\
0.042 \\
\end{array}$ & $\begin{array}{l}0.329 \\
0.413 \\
0.546 \\
0.462 \\
0.454 \\
0.470 \\
0.580 \\
1.367\end{array}$ & $\begin{array}{r}36 \\
22 \\
16 \\
11 \\
17 \\
4 \\
1 \\
-\end{array}$ & $\begin{array}{r}24 \\
47 \\
77 \\
79 \\
80 \\
49 \\
27 \\
4\end{array}$ & $\begin{array}{l}0.017 \\
0.013 \\
0.015 \\
0.016 \\
0.027 \\
0.009 \\
0.007\end{array}$ & $\begin{array}{l}0.027 \\
0.024 \\
0.031 \\
0.030 \\
0.030 \\
0.026 \\
0.040 \\
0.040\end{array}$ & $\begin{array}{r}17.0 \\
10.8 \\
18.6 \\
15.4 \\
21.1 \\
15.5 \\
6.0 \\
-\end{array}$ & $\begin{array}{l}12 \cdot 4 \\
17 \cdot 3 \\
17 \cdot 3 \\
15 \cdot 3 \\
15 \cdot 2 \\
18 \cdot 2 \\
14 \cdot 4 \\
34 \cdot 0\end{array}$ \\
\hline over) & - & 15 & - & - & - & - & - & - & - & - & - & - \\
\hline Total & 6,788 & 13,295 & 1,743 & 6,286 & - & - & 107 & 387 & - & - & - & - \\
\hline
\end{tabular}

TABLE 7: BROAD DIAGNOSTIC GROUP 6: DISEASES OF THE EYE (I.S.C. Nos. 370-389)

\begin{tabular}{|c|c|c|c|c|c|c|c|c|c|c|c|c|}
\hline \multirow[t]{2}{*}{ Age Group } & \multicolumn{2}{|c|}{$\begin{array}{l}\text { Exposure to Risk } \\
\text { (Man Years) }\end{array}$} & \multicolumn{2}{|c|}{$\begin{array}{c}\text { No. of Days } \\
\text { Sickness Absence* }\end{array}$} & \multicolumn{2}{|c|}{$\begin{array}{c}\text { Average Annual } \\
\text { Duration Per Person } \\
\text { (Days) }\end{array}$} & \multicolumn{2}{|c|}{ No. of Spells } & \multicolumn{2}{|c|}{$\begin{array}{c}\text { Annual Inception } \\
\text { Rate } \\
\text { (Spells) }\end{array}$} & \multicolumn{2}{|c|}{$\begin{array}{l}\text { Average Length } \\
\text { of Spell } \\
\text { (Days) }\end{array}$} \\
\hline & Single & Married & Single & Married & Single & Married & Single & Married & Single & Married & Single & Married \\
\hline $\begin{array}{l}\text { Up to } 24 \\
25-29 \\
30-34 \\
35-39 \\
40-44 \\
45-49 \\
50-54 \\
55-59 \\
60-a n d\end{array}$ & $\begin{array}{r}2,100 \\
1,668 \\
1,082 \\
694 \\
625 \\
460 \\
142 \\
17\end{array}$ & $\begin{array}{r}904 \\
1,971 \\
2,447 \\
2,611 \\
2,679 \\
1,898 \\
670 \\
100\end{array}$ & $\begin{array}{r}231 \\
44 \\
107 \\
42 \\
43 \\
20 \\
121\end{array}$ & $\begin{array}{r}82 \\
181 \\
420 \\
184 \\
301 \\
502 \\
179 \\
18\end{array}$ & $\begin{array}{r}0.110 \\
0.026 \\
0.099 \\
0.060 \\
0.069 \\
0.043 \\
0.855 \\
-\end{array}$ & $\begin{array}{l}0.091 \\
0.092 \\
0.172 \\
0.070 \\
0 \cdot 112 \\
0.265 \\
0 \cdot 267 \\
0.181\end{array}$ & $\begin{array}{r}14 \\
5 \\
12 \\
5 \\
6 \\
3 \\
2 \\
-\end{array}$ & $\begin{array}{r}6 \\
16 \\
25 \\
23 \\
25 \\
16 \\
8 \\
3\end{array}$ & $\begin{array}{l}0.007 \\
0.003 \\
0.011 \\
0.007 \\
0.010 \\
0.007 \\
0.014\end{array}$ & $\begin{array}{l}0.007 \\
0.008 \\
0.010 \\
0.009 \\
0.009 \\
0.008 \\
0.012 \\
0.030\end{array}$ & $\begin{array}{r}16 \cdot 5 \\
8 \cdot 8 \\
8.9 \\
8 \cdot 4 \\
7 \cdot 2 \\
6 \cdot 7 \\
60 \cdot 5 \\
-\end{array}$ & $\begin{array}{r}13 \cdot 7 \\
11 \cdot 3 \\
16 \cdot 8 \\
8 \cdot 0 \\
12 \cdot 0 \\
31 \cdot 4 \\
22 \cdot 4 \\
6.0\end{array}$ \\
\hline over) & - & 15 & - & - & - & - & - & - & - & - & - & - \\
\hline Total & 6,788 & 13,295 & 608 & 1,867 & - & - & 47 & 122 & - & - & - & - \\
\hline
\end{tabular}

*Excludes days beyond first six months of absence. 
TABLE 8: BROAD DIAGNOSTIC GROUP 7: DISEASES OF THE EAR (I.S.C. Nos. 390-398)

\begin{tabular}{|c|c|c|c|c|c|c|c|c|c|c|c|c|}
\hline \multirow[t]{2}{*}{ Age Group } & \multicolumn{2}{|c|}{$\begin{array}{l}\text { Exposure to Risk } \\
\text { (Man Years) }\end{array}$} & \multicolumn{2}{|c|}{$\begin{array}{c}\text { No. of Days } \\
\text { Sickness Absence* }\end{array}$} & \multicolumn{2}{|c|}{$\begin{array}{c}\text { Average Annual } \\
\text { Duration Per Person } \\
\text { (Days) }\end{array}$} & \multicolumn{2}{|c|}{ No. of Spells } & \multicolumn{2}{|c|}{$\begin{array}{c}\text { Annual Inception } \\
\text { Rate } \\
\text { (Spells) }\end{array}$} & \multicolumn{2}{|c|}{$\begin{array}{l}\text { Average Length } \\
\text { of Spell } \\
\text { (Days) }\end{array}$} \\
\hline & Single & Married & Single & Married & Single & Married & Single & Married & Single & Married & Single & Married \\
\hline $\begin{array}{l}\text { Up to } 24 \\
25-29 \\
30-34 \\
35-39 \\
40-44 \\
45-49 \\
50-54 \\
55-59 \\
\text { (60 and } \\
\text { over) }\end{array}$ & $\begin{array}{r}2,100 \\
1,668 \\
1,082 \\
694 \\
625 \\
460 \\
142 \\
17 \\
-\end{array}$ & $\begin{array}{r}904 \\
1,971 \\
2,447 \\
2,611 \\
2,679 \\
1,898 \\
670 \\
100 \\
15\end{array}$ & $\begin{array}{r}63 \\
353 \\
12 \\
45 \\
97 \\
84 \\
- \\
- \\
-\end{array}$ & $\begin{array}{r}81 \\
251 \\
252 \\
276 \\
354 \\
85 \\
36 \\
13 \\
-\end{array}$ & $\begin{array}{r}0.030 \\
0.211 \\
0.011 \\
0.065 \\
0.155 \\
0.183 \\
- \\
- \\
-\end{array}$ & $\begin{array}{r}0 \cdot 090 \\
0 \cdot 127 \\
0 \cdot 103 \\
0 \cdot 106 \\
0 \cdot 132 \\
0 \cdot 045 \\
0.054 \\
0 \cdot 131 \\
-\end{array}$ & $\begin{array}{r}9 \\
16 \\
1 \\
2 \\
4 \\
3 \\
- \\
- \\
-\end{array}$ & $\begin{array}{r}6 \\
19 \\
20 \\
19 \\
21 \\
9 \\
1 \\
1 \\
-\end{array}$ & $\begin{array}{r}0.004 \\
0.010 \\
0.001 \\
0.003 \\
0.006 \\
0.007 \\
- \\
- \\
-\end{array}$ & $\begin{array}{r}0.007 \\
0.010 \\
0.008 \\
0.007 \\
0.008 \\
0.005 \\
0.001 \\
0.010 \\
-\end{array}$ & $\begin{array}{r}7 \cdot 0 \\
22 \cdot 0 \\
12 \cdot 0 \\
22 \cdot 5 \\
24 \cdot 3 \\
28 \cdot 0 \\
- \\
- \\
-\end{array}$ & $\begin{array}{r}13.5 \\
13.2 \\
12.6 \\
14.5 \\
16.9 \\
9.4 \\
36.0 \\
13.0 \\
-\end{array}$ \\
\hline Toral & 6,788 & 13,295 & 653 & 1,348 & - & - & 35 & 96 & - & - & - & 一 \\
\hline
\end{tabular}

TABLE 9: BROAD DIAGNOSTIC GROUP 8: DISEASES OF THE CIRCULATORY SYSTEM (I.S.C. Nos. 400-468, 782)

\begin{tabular}{|c|c|c|c|c|c|c|c|c|c|c|c|c|}
\hline \multirow[t]{2}{*}{ Age Group } & \multicolumn{2}{|c|}{$\begin{array}{c}\text { Exposure to Risk } \\
\text { (Man Years) }\end{array}$} & \multicolumn{2}{|c|}{$\begin{array}{c}\text { No. of Days } \\
\text { Sickness Absence* }\end{array}$} & \multicolumn{2}{|c|}{$\begin{array}{c}\text { Average Annual } \\
\text { Duration Per Person } \\
\text { (Days) }\end{array}$} & \multicolumn{2}{|c|}{ No. of Spells } & \multicolumn{2}{|c|}{$\begin{array}{c}\text { Annual Inception } \\
\text { Rate } \\
\text { (Spells) }\end{array}$} & \multicolumn{2}{|c|}{$\begin{array}{l}\text { Average Length } \\
\text { of Spell } \\
\text { (Days) }\end{array}$} \\
\hline & Single & Married & Single & Married & Single & Married & Single & Married & Single & Married & Single & Married \\
\hline $\begin{array}{l}\text { Up to } 24 \\
25-29 \\
30-34 \\
35-39 \\
40-44 \\
45-49 \\
50-54 \\
55-59 \\
(60 \text { and }\end{array}$ & $\begin{array}{r}2,100 \\
1,668 \\
1,082 \\
694 \\
625 \\
460 \\
142 \\
17\end{array}$ & $\begin{array}{r}904 \\
1,971 \\
2,447 \\
2,611 \\
2,679 \\
1,898 \\
670 \\
100\end{array}$ & $\begin{array}{r}334 \\
461 \\
107 \\
217 \\
162 \\
100 \\
11 \\
\end{array}$ & $\begin{array}{r}190 \\
605 \\
1,021 \\
1,423 \\
1,663 \\
1,162 \\
1,224 \\
188\end{array}$ & $\begin{array}{l}0.159 \\
0.276 \\
0.099 \\
0.312 \\
0.259 \\
0.217 \\
0.078\end{array}$ & $\begin{array}{l}0.210 \\
0.307 \\
0.418 \\
0.545 \\
0.620 \\
0.612 \\
1.835 \\
1.889\end{array}$ & $\begin{array}{r}20 \\
21 \\
8 \\
5 \\
8 \\
9 \\
1 \\
-\end{array}$ & $\begin{array}{r}14 \\
25 \\
46 \\
44 \\
45 \\
36 \\
30 \\
5\end{array}$ & $\begin{array}{r}0.010 \\
0.013 \\
0.007 \\
0.007 \\
0.013 \\
0.020 \\
0.007 \\
-\end{array}$ & $\begin{array}{l}0.015 \\
0.013 \\
0.019 \\
0.017 \\
0.017 \\
0.019 \\
0.045 \\
0.050\end{array}$ & $\begin{array}{l}16 \cdot 7 \\
22 \cdot 0 \\
13 \cdot 4 \\
43 \cdot 4 \\
20 \cdot 3 \\
11 \cdot 1 \\
11 \cdot 0 \\
-\end{array}$ & $\begin{array}{l}13 \cdot 6 \\
24 \cdot 2 \\
22 \cdot 2 \\
32 \cdot 3 \\
37 \cdot 0 \\
32 \cdot 3 \\
40 \cdot 8 \\
37 \cdot 6\end{array}$ \\
\hline over) & 一 & 15 & - & - & - & 一 & - & - & - & - & - & - \\
\hline Total & 6,788 & 13,295 & 1,392 & 7,476 & - & - & 72 & 245 & - & - & - & - \\
\hline
\end{tabular}

TABLE 10: BROAD DIAGNOSTIC GROUP 9: COLDS AND INFLUENZA (I.S.C. Nos. 470, 480-483)

\begin{tabular}{|c|c|c|c|c|c|c|c|c|c|c|c|c|}
\hline \multirow[t]{2}{*}{ Age Group } & \multicolumn{2}{|c|}{$\begin{array}{l}\text { Exposure to Risk } \\
\text { (Man Years) }\end{array}$} & \multicolumn{2}{|c|}{$\begin{array}{c}\text { No. of Days } \\
\text { Sickness Absence* }\end{array}$} & \multicolumn{2}{|c|}{$\begin{array}{c}\text { Average Annual } \\
\text { Duration Per Person } \\
\text { (Days) }\end{array}$} & \multicolumn{2}{|c|}{ No. of Spells } & \multicolumn{2}{|c|}{$\begin{array}{c}\text { Annual Inception } \\
\text { Rate } \\
\text { (Spells) }\end{array}$} & \multicolumn{2}{|c|}{$\begin{array}{l}\text { Average Length } \\
\text { of Spell } \\
\text { (Days) }\end{array}$} \\
\hline & Single & Married & Single & Married & Single & Married & Single & Married & Single & Married & Single & Married \\
\hline $\begin{array}{l}\text { Up to } 24 \\
25-29 \\
30-34 \\
35-39 \\
40-44 \\
45-49 \\
50-54 \\
55-59 \\
\text { (60 and } \\
\text { over) }\end{array}$ & $\begin{array}{r}2,100 \\
1,668 \\
1,082 \\
694 \\
625 \\
460 \\
142 \\
17\end{array}$ & $\begin{array}{r}904 \\
1,971 \\
2,447 \\
2,611 \\
2,679 \\
1,898 \\
670 \\
100 \\
15\end{array}$ & $\begin{array}{r}5,066 \\
4,297 \\
2,971 \\
1,457 \\
1,424 \\
1,458 \\
270 \\
39 \\
-\end{array}$ & $\begin{array}{r}3,599 \\
7,373 \\
8,974 \\
10,944 \\
9,771 \\
6,963 \\
3,004 \\
456 \\
55\end{array}$ & $\begin{array}{l}2.411 \\
2.574 \\
2.745 \\
2.098 \\
2.278 \\
3.170 \\
1.908 \\
2 \cdot 294\end{array}$ & $\begin{array}{l}3.984 \\
3 \cdot 738 \\
3 \cdot 670 \\
4 \cdot 192 \\
3.645 \\
3.670 \\
4 \cdot 479 \\
4.583 \\
3.667\end{array}$ & $\begin{array}{r}559 \\
430 \\
262 \\
129 \\
121 \\
105 \\
21 \\
3 \\
-\end{array}$ & $\begin{array}{r}347 \\
682 \\
760 \\
893 \\
752 \\
513 \\
218 \\
33 \\
4\end{array}$ & $\begin{array}{r}0 \cdot 266 \\
0 \cdot 258 \\
0 \cdot 242 \\
0 \cdot 186 \\
0 \cdot 194 \\
0 \cdot 228 \\
0 \cdot 148 \\
0 \cdot 176 \\
-\end{array}$ & $\begin{array}{l}0.384 \\
0.346 \\
0.311 \\
0.342 \\
0.280 \\
0.270 \\
0.325 \\
0.332 \\
0.267\end{array}$ & $\begin{array}{r}9.1 \\
10.0 \\
11.3 \\
11.3 \\
11.8 \\
13.9 \\
12.9 \\
13.0 \\
-\end{array}$ & $\begin{array}{l}10.4 \\
10.8 \\
11.8 \\
12.3 \\
13.0 \\
13.6 \\
13.8 \\
13.8 \\
13.8\end{array}$ \\
\hline Total & 6,788 & 13,295 & 16,982 & 51,139 & - & - & 1,630 & 4,202 & - & - & - & - \\
\hline
\end{tabular}

TABLE 11: BROAD DIAGNOSTIC GROUP 10: BRONCHITIS (I.S.C. Nos. 500-502)

\begin{tabular}{|c|c|c|c|c|c|c|c|c|c|c|c|c|}
\hline \multirow[t]{2}{*}{ Age Group } & \multicolumn{2}{|c|}{$\begin{array}{l}\text { Exposure to Risk } \\
\text { (Man Years) }\end{array}$} & \multicolumn{2}{|c|}{$\begin{array}{c}\text { No. of Days } \\
\text { Sickness Absence* }\end{array}$} & \multicolumn{2}{|c|}{$\begin{array}{c}\text { Average Annual } \\
\text { Duration Per Person } \\
\text { (Days) }\end{array}$} & \multicolumn{2}{|c|}{ No. of Spells } & \multicolumn{2}{|c|}{$\begin{array}{c}\text { Annual Inception } \\
\text { Rate } \\
\text { (Spells) }\end{array}$} & \multicolumn{2}{|c|}{$\begin{array}{l}\text { Average Length } \\
\text { of Spell } \\
\text { (Days) }\end{array}$} \\
\hline & Single & Married & Single & Married & Single & Married & Single & Married & Single & Married & Single & Married \\
\hline \multirow[t]{2}{*}{$\begin{array}{l}\text { Up to } 24 \\
25-29 \\
30-34 \\
35-39 \\
40-44 \\
45-49 \\
50-54 \\
55-59 \\
\text { (60 and } \\
\text { over) }\end{array}$} & $\begin{array}{r}2,100 \\
1,668 \\
1,082 \\
694 \\
625 \\
460 \\
142 \\
17\end{array}$ & $\begin{array}{r}904 \\
1,971 \\
2,447 \\
2,611 \\
2,679 \\
1,898 \\
670 \\
100\end{array}$ & $\begin{array}{r}1,694 \\
1,815 \\
1,180 \\
1,010 \\
913 \\
761 \\
532 \\
18\end{array}$ & $\begin{array}{r}1,057 \\
2,634 \\
3,683 \\
3,704 \\
5,093 \\
3,652 \\
2,358 \\
412\end{array}$ & $\begin{array}{l}0.806 \\
1.087 \\
1.090 \\
1.454 \\
1.461 \\
1.654 \\
3.760 \\
1.059\end{array}$ & $\begin{array}{l}1.170 \\
1.335 \\
1.506 \\
1.419 \\
1.900 \\
1.925 \\
3.516 \\
4.141\end{array}$ & $\begin{array}{r}130 \\
131 \\
82 \\
50 \\
59 \\
29 \\
14 \\
1\end{array}$ & $\begin{array}{r}80 \\
198 \\
252 \\
275 \\
310 \\
200 \\
105 \\
25\end{array}$ & $\begin{array}{l}0.062 \\
0.078 \\
0.076 \\
0.072 \\
0.094 \\
0.063 \\
0.099 \\
0.059\end{array}$ & $\begin{array}{l}0.089 \\
0 \cdot 100 \\
0 \cdot 103 \\
0 \cdot 105 \\
0 \cdot 116 \\
0 \cdot 105 \\
0 \cdot 157 \\
0 \cdot 251\end{array}$ & $\begin{array}{l}13.0 \\
13.9 \\
14.4 \\
20.2 \\
15.5 \\
26.2 \\
38.0 \\
18.0\end{array}$ & $\begin{array}{l}13.2 \\
13.3 \\
14.6 \\
13.5 \\
16.4 \\
18.3 \\
22.5 \\
16.5\end{array}$ \\
\hline & - & 15 & - & 184 & - & $12 \cdot 267$ & - & 5 & - & 0.333 & - & $36 \cdot 8$ \\
\hline Total & 6,788 & 13,295 & 7,923 & 22,777 & - & - & 496 & 1,450 & - & - & - & - \\
\hline
\end{tabular}

*Excludes days beyond first six months of absence. 
TABLE 12: BROAD DIAGNOSTIC GROUP 11: OTHER RESPIRATORY DISEASES (I.S.C. Nos. 471-475, 490-493, 510-527, 763, 783)

\begin{tabular}{|c|c|c|c|c|c|c|c|c|c|c|c|c|}
\hline \multirow[t]{2}{*}{ Age Group } & \multicolumn{2}{|c|}{$\begin{array}{l}\text { Exposure to Risk } \\
\text { (Man Years) }\end{array}$} & \multicolumn{2}{|c|}{$\begin{array}{l}\text { No. of Days } \\
\text { Sickness Absence* }\end{array}$} & \multicolumn{2}{|c|}{$\begin{array}{c}\text { Average Annual } \\
\text { Duration Per Person } \\
\text { (Days) }\end{array}$} & \multicolumn{2}{|c|}{ No. of Spells } & \multicolumn{2}{|c|}{$\begin{array}{c}\text { Annual Inception } \\
\text { Rate } \\
\text { (Spells) }\end{array}$} & \multicolumn{2}{|c|}{$\begin{array}{l}\text { Average Length } \\
\text { of Spell } \\
\text { (Days) }\end{array}$} \\
\hline & Single & Married & Single & Married & Single & Married & Single & Married & Single & Married & Single & Married \\
\hline $\begin{array}{l}\text { Up to } 24 \\
25-29 \\
30-34 \\
35-39 \\
40-44 \\
45-49 \\
50-54 \\
55-59 \\
\text { (60 and }\end{array}$ & $\begin{array}{r}2,100 \\
1,668 \\
1,082 \\
694 \\
625 \\
460 \\
142 \\
17\end{array}$ & $\begin{array}{r}904 \\
1,971 \\
2,447 \\
2,611 \\
2,679 \\
1,898 \\
670 \\
100\end{array}$ & $\begin{array}{r}3,048 \\
2,455 \\
1,556 \\
724 \\
1,080 \\
532 \\
223 \\
39\end{array}$ & $\begin{array}{r}1,762 \\
3,531 \\
3,506 \\
4,021 \\
4,186 \\
3,400 \\
1,099 \\
401\end{array}$ & $\begin{array}{l}1.451 \\
1.471 \\
1.438 \\
1.043 \\
1.728 \\
1.157 \\
1.576 \\
2.294\end{array}$ & $\begin{array}{l}1.951 \\
1.790 \\
1.434 \\
1.540 \\
1.561 \\
1.792 \\
1.639 \\
4.030\end{array}$ & $\begin{array}{r}266 \\
208 \\
104 \\
53 \\
46 \\
34 \\
14 \\
2\end{array}$ & $\begin{array}{r}147 \\
289 \\
294 \\
273 \\
243 \\
174 \\
64 \\
12\end{array}$ & $\begin{array}{l}0.127 \\
0.125 \\
0.096 \\
0.076 \\
0.074 \\
0.074 \\
0.099 \\
0.118\end{array}$ & $\begin{array}{l}0.163 \\
0.147 \\
0.120 \\
0.105 \\
0.091 \\
0.092 \\
0.095 \\
0.121\end{array}$ & $\begin{array}{l}11.5 \\
11.8 \\
15.0 \\
13.7 \\
23.5 \\
15.6 \\
15.9 \\
19.5\end{array}$ & $\begin{array}{l}12.0 \\
12.2 \\
11.9 \\
14 \cdot 7 \\
17.2 \\
19.5 \\
17.2 \\
33.4\end{array}$ \\
\hline over) & - & 15 & - & - & - & - & - & - & - & - & - & - \\
\hline Total & 6,788 & 13,295 & 9,657 & 21,906 & - & - & 727 & 1,496 & - & - & - & - \\
\hline
\end{tabular}

TABLE 13: BROAD DIAGNOSTIC GROUP 12: DISEASES OF STOMACH AND DUODENUM (I.S.C. Nos. 540-545, 784)

\begin{tabular}{|c|c|c|c|c|c|c|c|c|c|c|c|c|}
\hline \multirow[t]{2}{*}{ Age Group } & \multicolumn{2}{|c|}{$\begin{array}{c}\text { Exposure to Risk } \\
\text { (Man Years) }\end{array}$} & \multicolumn{2}{|c|}{$\begin{array}{l}\text { No. of Days } \\
\text { Sickness Absence* }\end{array}$} & \multicolumn{2}{|c|}{\begin{tabular}{|} 
Average Annual \\
Duration Per Person \\
(Days)
\end{tabular}} & \multicolumn{2}{|c|}{ No. of Spells } & \multicolumn{2}{|c|}{$\begin{array}{c}\text { Annual Inception } \\
\text { Rate } \\
\text { (Spells) }\end{array}$} & \multicolumn{2}{|c|}{$\begin{array}{l}\text { Average Length } \\
\text { of Spell } \\
\text { (Days) }\end{array}$} \\
\hline & Single & Married & Single & Married & Single & Married & Single & Married & Single & Married & Single & Married \\
\hline \multirow[t]{2}{*}{$\begin{array}{l}\text { Up to } 24 \\
25-29 \\
30-34 \\
35-39 \\
40-44 \\
45-49 \\
50-54 \\
55-59 \\
\text { (60 and } \\
\text { over) }\end{array}$} & $\begin{array}{r}2,100 \\
1,668 \\
1,082 \\
694 \\
625 \\
460 \\
142 \\
17\end{array}$ & $\begin{array}{r}904 \\
1,971 \\
2,447 \\
2,611 \\
2,679 \\
1,898 \\
670 \\
100\end{array}$ & $\begin{array}{r}1,726 \\
1,368 \\
1,273 \\
396 \\
577 \\
560 \\
107 \\
-\end{array}$ & $\begin{array}{r}1,193 \\
2,090 \\
2,711 \\
2,838 \\
3,077 \\
2,121 \\
580 \\
141\end{array}$ & $\begin{array}{l}0.822 \\
0.819 \\
1.176 \\
0.570 \\
0.923 \\
1.217 \\
0.756 \\
-\end{array}$ & $\begin{array}{l}1.321 \\
1.060 \\
1.109 \\
1.087 \\
1.148 \\
1.118 \\
0.865 \\
1.417\end{array}$ & $\begin{array}{r}122 \\
85 \\
76 \\
33 \\
37 \\
33 \\
5 \\
-\end{array}$ & $\begin{array}{r}102 \\
180 \\
175 \\
149 \\
164 \\
111 \\
27 \\
9\end{array}$ & $\begin{array}{l}0.058 \\
0.051 \\
0.070 \\
0.048 \\
0.059 \\
0.072 \\
0.035 \\
\end{array}$ & $\begin{array}{l}0.113 \\
0.091 \\
0.072 \\
0.057 \\
0.061 \\
0.058 \\
0.040 \\
0.090\end{array}$ & $\begin{array}{l}14 \cdot 1 \\
16.1 \\
16.8 \\
12.0 \\
15 \cdot 6 \\
17.0 \\
21.4\end{array}$ & $\begin{array}{l}11.7 \\
11.6 \\
15.5 \\
19.0 \\
18.8 \\
19.1 \\
21.5 \\
15.7\end{array}$ \\
\hline & - & 15 & - & 14 & - & 0.933 & - & 2 & - & 0.133 & - & 7.0 \\
\hline Total & 6,788 & 13,295 & 6,007 & 14,765 & - & - & 391 & 919 & - & - & - & - \\
\hline
\end{tabular}

TABLE 14: BROAD DiAgNOSTIC GROUP 13: HERNIA OF ABDOMINAL CAVITY (I.S.C. Nos. 560-561)

\begin{tabular}{|c|c|c|c|c|c|c|c|c|c|c|c|c|}
\hline \multirow[t]{2}{*}{ Age Group } & \multicolumn{2}{|c|}{$\begin{array}{c}\text { Exposure to Risk } \\
\text { (Man Years) }\end{array}$} & \multicolumn{2}{|c|}{$\begin{array}{c}\text { No. of Days } \\
\text { Sickness Absence* }\end{array}$} & \multicolumn{2}{|c|}{$\mid \begin{array}{c}\text { Average Annual } \\
\text { Duration Per Person } \\
\text { (Days) }\end{array}$} & \multicolumn{2}{|c|}{ No. of Spells } & \multicolumn{2}{|c|}{$\begin{array}{c}\text { Annual Inception } \\
\text { Rate } \\
\text { (Spells) }\end{array}$} & \multicolumn{2}{|c|}{$\begin{array}{l}\text { Average Length } \\
\text { of Spell } \\
\text { (Days) }\end{array}$} \\
\hline & Single & Married & Single & Married & Single & Married & Single & Married & Single & Married & Single & Married \\
\hline $\begin{array}{l}\text { Up to } 24 \\
25-29 \\
30-34 \\
35-39 \\
40-44 \\
45-49 \\
50-54 \\
55-59 \\
(60 \text { and } \\
\text { over) }\end{array}$ & $\begin{array}{r}2,100 \\
1,668 \\
1,082 \\
694 \\
625 \\
460 \\
142 \\
17 \\
-\end{array}$ & $\begin{array}{r}904 \\
1,971 \\
2,447 \\
2,611 \\
2,679 \\
1,898 \\
670 \\
100 \\
15\end{array}$ & $\begin{array}{l}\bar{z} \\
\bar{z} \\
\frac{\overrightarrow{166}}{-} \\
-\end{array}$ & $\begin{array}{r}15 \\
198 \\
275 \\
200 \\
532 \\
665 \\
99 \\
-\end{array}$ & $\begin{array}{l}\bar{z} \\
\bar{z} \\
\frac{361}{z} \\
-\end{array}$ & $\begin{array}{c}0.017 \\
0 \cdot 100 \\
0.112 \\
0.077 \\
0 \cdot 198 \\
0.350 \\
0.995 \\
-\end{array}$ & $\begin{array}{l}= \\
\bar{z} \\
\frac{1}{-} \\
-\end{array}$ & $\begin{array}{r}1 \\
6 \\
4 \\
4 \\
6 \\
11 \\
1 \\
-\end{array}$ & $\begin{array}{c}\bar{z} \\
\bar{z} \\
0 \cdot \overline{002} \\
\overline{-} \\
-\end{array}$ & $\begin{array}{c}0.001 \\
0.003 \\
0.002 \\
0.002 \\
0.002 \\
0.006 \\
0 . \overline{010} \\
-\end{array}$ & $\begin{array}{c}\bar{Z} \\
\bar{z} \\
166 \cdot 0 \\
= \\
-\end{array}$ & $\begin{array}{l}15 \cdot 0 \\
33 \cdot 0 \\
68.8 \\
50 \cdot 0 \\
88.7 \\
60 \cdot 5 \\
\overline{99 \cdot 0} \\
-\end{array}$ \\
\hline Total & 6,788 & 13,295 & 166 & 1,984 & - & - & 1 & 33 & - & - & - & - \\
\hline
\end{tabular}

TABLE 15: BROAD DIAGNOSTIC GROUP 14: OTHER DISEASES OF THE DIGESTIVE SYSTEM (I.S.C. Nos. 530-539, 550-553, 570-587, 764, 785)

\begin{tabular}{|c|c|c|c|c|c|c|c|c|c|c|c|c|}
\hline \multirow[t]{2}{*}{ Age Group } & \multicolumn{2}{|c|}{$\begin{array}{c}\text { Exposure to Risk } \\
\text { (Man Years) }\end{array}$} & \multicolumn{2}{|c|}{$\begin{array}{c}\text { No. of Days } \\
\text { Sickness Absence* }\end{array}$} & \multicolumn{2}{|c|}{$\begin{array}{c}\text { Average Annual } \\
\text { Duration Per Person } \\
\text { (Days) }\end{array}$} & \multicolumn{2}{|c|}{ No. of Spells } & \multicolumn{2}{|c|}{$\begin{array}{c}\text { Annual Inception } \\
\text { Rate } \\
\text { (Spells) }\end{array}$} & \multicolumn{2}{|c|}{$\begin{array}{c}\text { Average Length } \\
\text { of Spell } \\
\text { (Days) }\end{array}$} \\
\hline & Single & Married & Single & Married & Single & Married & Single & Married & Single & Married & Single & Married \\
\hline $\begin{array}{l}\text { Up to } 24 \\
25-29 \\
30-34 \\
35-39 \\
40-44 \\
45-49 \\
50-54 \\
55-59 \\
60 \text { and }\end{array}$ & $\begin{array}{r}2,100 \\
1,668 \\
1,082 \\
694 \\
625 \\
460 \\
142 \\
17\end{array}$ & $\begin{array}{r}904 \\
1,971 \\
2,447 \\
2,611 \\
2,679 \\
1,898 \\
670 \\
100\end{array}$ & $\begin{array}{r}2,213 \\
1,572 \\
838 \\
607 \\
389 \\
601 \\
64 \\
-\end{array}$ & $\begin{array}{r}1,154 \\
2,914 \\
3,139 \\
3,351 \\
2,472 \\
3,329 \\
866 \\
53\end{array}$ & $\begin{array}{c}1.053 \\
0.942 \\
0.774 \\
0.874 \\
0.622 \\
1.307 \\
0.452 \\
-\end{array}$ & $\begin{array}{l}1.277 \\
1.477 \\
1.284 \\
1.283 \\
0.922 \\
1.754 \\
1.291 \\
0.533\end{array}$ & $\begin{array}{r}149 \\
113 \\
69 \\
36 \\
30 \\
28 \\
6 \\
-\end{array}$ & $\begin{array}{r}83 \\
207 \\
218 \\
188 \\
169 \\
146 \\
32 \\
4\end{array}$ & $\begin{array}{l}0.071 \\
0.068 \\
0.064 \\
0.052 \\
0.048 \\
0.061 \\
0.042 \\
-\end{array}$ & $\begin{array}{l}0.092 \\
0.105 \\
0.089 \\
0.072 \\
0.063 \\
0.077 \\
0.048 \\
0.040\end{array}$ & $\begin{array}{l}14.9 \\
13.9 \\
12.1 \\
16.9 \\
13.0 \\
21.5 \\
10.7 \\
-\end{array}$ & $\begin{array}{l}13.9 \\
14.1 \\
14.4 \\
17.8 \\
14.6 \\
22.8 \\
27.1 \\
13.3\end{array}$ \\
\hline over) & - & 15 & - & - & - & - & - & - & - & - & - & - \\
\hline Total & 6,788 & 13,295 & 6,284 & $\mid 17,278$ & - & - & 431 & 1,047 & - & - & - & - \\
\hline
\end{tabular}

*Excludes days beyond first six months of absence. 
TABLE 16: BROAD DIAGNOSTIC GROUP 15: DISEASES OF WOMEN (I.S.C. Nos. 620-689)

\begin{tabular}{|c|c|c|c|c|c|c|c|c|c|c|c|c|}
\hline \multirow[t]{2}{*}{ Age Group } & \multicolumn{2}{|c|}{$\begin{array}{l}\text { Exposure to Risk } \\
\text { (Man Years) }\end{array}$} & \multicolumn{2}{|c|}{$\begin{array}{l}\text { No. of Days } \\
\text { Sickness Absence* }\end{array}$} & \multicolumn{2}{|c|}{$\mid$\begin{tabular}{c|} 
Average Annual \\
Duration Per Person \\
(Days)
\end{tabular}} & \multicolumn{2}{|c|}{ No. of Spells } & \multicolumn{2}{|c|}{$\begin{array}{c}\text { Annual Inception } \\
\text { Rate } \\
\text { (Spells) }\end{array}$} & \multicolumn{2}{|c|}{$\begin{array}{l}\text { Average Length } \\
\text { of Spell } \\
\text { (Days) }\end{array}$} \\
\hline & Single & Married & Single & Married & Single & Married & Single & Married & Single & Married & Single & Married \\
\hline $\begin{array}{l}\text { Up to } 24 \\
25-29 \\
30-34 \\
35-39 \\
40-44 \\
45-49 \\
50-54 \\
55-59 \\
\text { (60 and } \\
\text { over) }\end{array}$ & $\begin{array}{r}2,100 \\
1,668 \\
1,082 \\
694 \\
625 \\
460 \\
142 \\
17 \\
-\end{array}$ & $\begin{array}{r}904 \\
1,971 \\
2,447 \\
2,611 \\
2,679 \\
1,898 \\
670 \\
100 \\
15\end{array}$ & $\begin{array}{r}1,073 \\
1,008 \\
727 \\
933 \\
183 \\
68 \\
138 \\
- \\
-\end{array}$ & $\begin{array}{r}1,832 \\
4,310 \\
4,414 \\
4,018 \\
6,003 \\
3,872 \\
1,113 \\
24 \\
-\end{array}$ & $\begin{array}{c}0.511 \\
0.604 \\
0.672 \\
1.344 \\
0.293 \\
0.148 \\
0.975 \\
- \\
-\end{array}$ & $\begin{array}{c}2.028 \\
2 \cdot 185 \\
1.805 \\
1.539 \\
2.239 \\
2.041 \\
1.659 \\
0.241 \\
-\end{array}$ & $\begin{array}{r}56 \\
43 \\
27 \\
22 \\
8 \\
6 \\
3 \\
- \\
-\end{array}$ & $\begin{array}{r}74 \\
187 \\
171 \\
152 \\
134 \\
103 \\
26 \\
2 \\
-\end{array}$ & $\begin{array}{c}0.027 \\
0.026 \\
0.025 \\
0.032 \\
0.013 \\
0.013 \\
0.021 \\
- \\
-\end{array}$ & $\begin{array}{l}0.082 \\
0.095 \\
0.070 \\
0.058 \\
0.050 \\
0.054 \\
0.039 \\
0.020 \\
-\end{array}$ & $\begin{array}{l}19 \cdot 2 \\
23.4 \\
26.9 \\
42.4 \\
22.9 \\
11.3 \\
46.0 \\
- \\
-\end{array}$ & $\begin{array}{l}24 \cdot 8 \\
23.0 \\
25 \cdot 8 \\
26.4 \\
44.8 \\
37.6 \\
42.8 \\
12.0 \\
-\end{array}$ \\
\hline Total & 6,788 & 13,295 & 4,130 & 25,586 & - & - & 165 & 849 & - & - & - & - \\
\hline
\end{tabular}

TABLE 17: BROAD DIAGNOSTIC GROUP 16: DISEASES OF THE SKIN (I.S.C. Nos. 690-698, 700-716)

\begin{tabular}{|c|c|c|c|c|c|c|c|c|c|c|c|c|}
\hline \multirow[t]{2}{*}{ Age Group } & \multicolumn{2}{|c|}{$\begin{array}{c}\text { Exposure to Risk } \\
\text { (Man Years) }\end{array}$} & \multicolumn{2}{|c|}{$\begin{array}{c}\text { No. of Days } \\
\text { Sickness Absence* }\end{array}$} & \multicolumn{2}{|c|}{$\begin{array}{c}\text { Average Annual } \\
\text { Duration Per Person } \\
\text { (Days) }\end{array}$} & \multicolumn{2}{|c|}{ No. of Spells } & \multicolumn{2}{|c|}{$\begin{array}{c}\text { Annual Inception } \\
\text { Rate } \\
\text { (Spells) }\end{array}$} & \multicolumn{2}{|c|}{$\begin{array}{l}\text { Average Length } \\
\text { of Spell } \\
\text { (Days) }\end{array}$} \\
\hline & Single & Married & Single & Married & Single & Married & Single & Married & Single & Married & Single & Married \\
\hline $\begin{array}{l}\text { Up to } 24 \\
25-29 \\
30-34 \\
35-39 \\
40-44 \\
45-49 \\
50-54 \\
55-59 \\
(60 \text { and }\end{array}$ & $\begin{array}{r}2,100 \\
1,668 \\
1,082 \\
694 \\
625 \\
460 \\
142 \\
17\end{array}$ & $\begin{array}{r}904 \\
1,971 \\
2,447 \\
2,611 \\
2,679 \\
1,898 \\
670 \\
100\end{array}$ & $\begin{array}{c}779 \\
565 \\
350 \\
314 \\
280 \\
341 \\
51 \\
-\end{array}$ & $\begin{array}{r}736 \\
986 \\
1,217 \\
1,029 \\
1,180 \\
1,035 \\
568 \\
117\end{array}$ & $\begin{array}{c}0 \cdot 371 \\
0 \cdot 338 \\
0 \cdot 323 \\
0 \cdot 452 \\
0.448 \\
0 \cdot 741 \\
0 \cdot 360 \\
-\end{array}$ & $\begin{array}{l}0.815 \\
0 \cdot 500 \\
0.498 \\
0.394 \\
0.440 \\
0.545 \\
0.847 \\
1 \cdot 176\end{array}$ & $\begin{array}{r}73 \\
53 \\
30 \\
17 \\
19 \\
18 \\
5 \\
-\end{array}$ & $\begin{array}{r}37 \\
82 \\
90 \\
73 \\
71 \\
67 \\
20 \\
4\end{array}$ & $\begin{array}{c}0.035 \\
0.032 \\
0.028 \\
0.024 \\
0.030 \\
0.039 \\
0.035 \\
-\end{array}$ & $\begin{array}{l}0.041 \\
0.042 \\
0.037 \\
0.028 \\
0.026 \\
0.035 \\
0.030 \\
0.040\end{array}$ & $\begin{array}{l}10 \cdot 7 \\
10 \cdot 7 \\
11.7 \\
18.5 \\
14.7 \\
18.9 \\
10 \cdot 2 \\
-\end{array}$ & $\begin{array}{l}19.9 \\
12.0 \\
13.5 \\
14.1 \\
16.6 \\
15.4 \\
28.4 \\
29.3\end{array}$ \\
\hline over) & - & 15 & - & 50 & - & $3 \cdot 333$ & - & 2 & - & $0 \cdot 133$ & - & $25 \cdot 0$ \\
\hline Total & 6,788 & 13,295 & 2,680 & 6,918 & - & - & 215 & 446 & - & - & - & - \\
\hline
\end{tabular}

TABLE 18: BROAD DIAGNOSTIC GROUP 17: DISEASES OF BONES AND ORGANS OF MOVEMENT (I.S.C. Nos. 720-749, 787)

\begin{tabular}{|c|c|c|c|c|c|c|c|c|c|c|c|c|}
\hline \multirow[t]{2}{*}{ Age Group } & \multicolumn{2}{|c|}{$\begin{array}{l}\text { Exposure to Risk } \\
\text { (Man Years) }\end{array}$} & \multicolumn{2}{|c|}{$\begin{array}{c}\text { No. of Days } \\
\text { Sickness Absence* }\end{array}$} & \multicolumn{2}{|c|}{$\begin{array}{c}\text { Average Annual } \\
\text { Duration Per Person } \\
\text { (Days) }\end{array}$} & \multicolumn{2}{|c|}{ No. of Spells } & \multicolumn{2}{|c|}{$\begin{array}{c}\text { Annual Inception } \\
\text { Rate } \\
\text { (Spells) }\end{array}$} & \multicolumn{2}{|c|}{$\begin{array}{l}\text { Average Length } \\
\text { of Spell } \\
\text { (Days) }\end{array}$} \\
\hline & Single & Married & Single & Married & Single & Married & Single & Married & Single & Married & Single & Married \\
\hline \multirow[t]{2}{*}{$\begin{array}{l}\text { Up to } 24 \\
25-29 \\
30-34 \\
35-39 \\
40-44 \\
45-49 \\
50-54 \\
55-59 \\
\text { (60 and } \\
\text { over) }\end{array}$} & $\begin{array}{r}2,100 \\
1,668 \\
1,082 \\
694 \\
625 \\
460 \\
142 \\
17\end{array}$ & $\begin{array}{r}904 \\
1,971 \\
2,447 \\
2,611 \\
2,679 \\
1,898 \\
670 \\
100\end{array}$ & $\begin{array}{r}1,758 \\
2,074 \\
937 \\
1,407 \\
1,082 \\
1,122 \\
353 \\
112\end{array}$ & $\begin{array}{r}1,059 \\
2,975 \\
4,378 \\
4,735 \\
7,030 \\
6,341 \\
2,404 \\
317\end{array}$ & $\begin{array}{l}0.837 \\
1.242 \\
0.866 \\
2.026 \\
1.731 \\
2.439 \\
2.495 \\
6.588\end{array}$ & $\begin{array}{l}1 \cdot 172 \\
1.508 \\
1.791 \\
1.814 \\
2.622 \\
3.342 \\
3.584 \\
3.186\end{array}$ & $\begin{array}{r}111 \\
121 \\
67 \\
66 \\
66 \\
52 \\
14 \\
1\end{array}$ & $\begin{array}{r}87 \\
191 \\
243 \\
264 \\
341 \\
289 \\
104 \\
18\end{array}$ & $\begin{array}{l}0.053 \\
0.072 \\
0.062 \\
0.095 \\
0.106 \\
0.113 \\
0.099 \\
0.059\end{array}$ & $\begin{array}{l}\cdot 096 \\
0 \cdot 097 \\
0 \cdot 099 \\
0 \cdot 101 \\
0 \cdot 127 \\
0 \cdot 152 \\
0 \cdot 155 \\
0 \cdot 181\end{array}$ & $\begin{array}{r}15.8 \\
17.1 \\
14.0 \\
21.3 \\
16.4 \\
21.6 \\
25.2 \\
112.0\end{array}$ & $\begin{array}{l}12.2 \\
15.6 \\
18.0 \\
17.9 \\
20.6 \\
21.9 \\
23.1 \\
17.6\end{array}$ \\
\hline & - & 15 & - & 88 & - & 5.867 & - & 2 & - & 0.133 & - & $44 \cdot 0$ \\
\hline Total & 6,788 & 13,295 & 8,845 & 29,327 & - & - & 498 & 1,539 & - & - & - & - \\
\hline
\end{tabular}

TABLE 19: BROAD DIAGNOSTIC GROUP 18: ACCIDENTS ON DUTY (I.S.C. Nos. (N) 800-999)

\begin{tabular}{|c|c|c|c|c|c|c|c|c|c|c|c|c|}
\hline \multirow[t]{2}{*}{ Age Group } & \multicolumn{2}{|c|}{$\begin{array}{c}\text { Exposure to Risk } \\
\text { (Man Years) }\end{array}$} & \multicolumn{2}{|c|}{$\begin{array}{c}\text { No. of Days } \\
\text { Sickness Absence* }\end{array}$} & \multicolumn{2}{|c|}{$\begin{array}{c}\text { Average Annual } \\
\text { Duration Per Person } \\
\text { (Days) }\end{array}$} & \multicolumn{2}{|c|}{ No. of Spells } & \multicolumn{2}{|c|}{$\begin{array}{c}\text { Annual Inception } \\
\text { Rate } \\
\text { (Spells) }\end{array}$} & \multicolumn{2}{|c|}{$\begin{array}{l}\text { Average Length } \\
\text { of Spell } \\
\text { (Days) }\end{array}$} \\
\hline & Single & Married & Single & Married & Single & Married & Single & Married & Single & Married & Single & Married \\
\hline \multirow[t]{2}{*}{$\begin{array}{l}\text { Up to } 24 \\
25-29 \\
30-34 \\
35-39 \\
40-44 \\
45-49 \\
50-54 \\
55-59 \\
\text { (60 and } \\
\text { over) }\end{array}$} & $\begin{array}{r}2,100 \\
1,668 \\
1,082 \\
694 \\
625 \\
460 \\
142 \\
17\end{array}$ & $\begin{array}{r}904 \\
1,971 \\
2,447 \\
2,611 \\
2,679 \\
1,898 \\
670 \\
100\end{array}$ & $\begin{array}{r}1,782 \\
1,232 \\
904 \\
397 \\
641 \\
1,343 \\
227 \\
8\end{array}$ & $\begin{array}{r}1,048 \\
3,005 \\
2,974 \\
4,894 \\
4,497 \\
6,326 \\
1,596 \\
352\end{array}$ & $\begin{array}{l}0.848 \\
0.738 \\
0.834 \\
0.572 \\
1.026 \\
2.920 \\
1.604 \\
0.471\end{array}$ & $\begin{array}{l}1.160 \\
1.524 \\
1.216 \\
1.874 \\
1.677 \\
3.334 \\
2.380 \\
3.538\end{array}$ & $\begin{array}{r}112 \\
61 \\
45 \\
27 \\
30 \\
31 \\
6 \\
1\end{array}$ & $\begin{array}{r}61 \\
127 \\
139 \\
182 \\
177 \\
166 \\
50 \\
6\end{array}$ & $\begin{array}{l}0.053 \\
0.037 \\
0.042 \\
0.039 \\
0.048 \\
0.067 \\
0.042 \\
0.059\end{array}$ & $\begin{array}{l}0.068 \\
0.064 \\
0.057 \\
0.070 \\
0.066 \\
0.087 \\
0.075 \\
0.060\end{array}$ & $\begin{array}{l}15.9 \\
20.2 \\
20.1 \\
14.7 \\
21.4 \\
43.3 \\
37.8 \\
8.0\end{array}$ & $\begin{array}{c}17 \cdot 2 \\
23 \cdot 7 \\
21 \cdot 4 \\
26 \cdot 9 \\
25 \cdot 4 \\
38 \cdot 1 \\
31 \\
58 \cdot 7\end{array}$ \\
\hline & - & 15 & - & - & - & - & - & - & - & - & - & - \\
\hline Total & 6,788 & 13,295 & 6.534 & 24,692 & - & - & 313 & 908 & - & - & - & - \\
\hline
\end{tabular}

*Excludes days beyond first six months of absence. 
TABLE 20: BROAD DIAGNOSTIC GROUP 19: ACCIDENTS OFF DUTY (I.S.C. Nos. (N) 800-999)

\begin{tabular}{|c|c|c|c|c|c|c|c|c|c|c|c|c|}
\hline \multirow[t]{2}{*}{ Age Group } & \multicolumn{2}{|c|}{$\begin{array}{c}\text { Exposure to Risk } \\
\text { (Man Years) }\end{array}$} & \multicolumn{2}{|c|}{$\begin{array}{l}\text { No. of Days } \\
\text { Sickness Absence* }\end{array}$} & \multicolumn{2}{|c|}{\begin{tabular}{|} 
Average Annual \\
Duration Per Person \\
(Days)
\end{tabular}} & \multicolumn{2}{|c|}{ No. of Spells } & \multicolumn{2}{|c|}{$\begin{array}{c}\text { Annual Inception } \\
\text { Rate } \\
\text { (Spells) }\end{array}$} & \multicolumn{2}{|c|}{$\begin{array}{l}\text { Average Length } \\
\text { of Spell } \\
\text { (Days) }\end{array}$} \\
\hline & Single & Married & Single & Married & Single & Married & Single & Married & Single & Married & Single & Married \\
\hline $\begin{array}{l}\text { Up to } 24 \\
25-29 \\
30-34 \\
35-39 \\
40-44 \\
45-49 \\
50-54 \\
55-59\end{array}$ & $\begin{array}{r}2,100 \\
1,668 \\
1,082 \\
694 \\
625 \\
460 \\
142 \\
17\end{array}$ & $\begin{array}{r}904 \\
1,971 \\
2,447 \\
2,611 \\
2,679 \\
1,898 \\
670 \\
100\end{array}$ & $\begin{array}{r}1,028 \\
1,258 \\
660 \\
439 \\
520 \\
318 \\
87 \\
8\end{array}$ & $\begin{array}{r}770 \\
1,861 \\
2,445 \\
2,819 \\
3,263 \\
3,279 \\
1,190 \\
141\end{array}$ & $\begin{array}{l}0.489 \\
0.754 \\
0.610 \\
0.632 \\
0.832 \\
0.691 \\
0.615 \\
0.471\end{array}$ & $\begin{array}{l}0.852 \\
0.944 \\
1.000 \\
1.080 \\
1.217 \\
1.728 \\
1.774 \\
1.417\end{array}$ & $\begin{array}{r}78 \\
71 \\
43 \\
28 \\
22 \\
17 \\
4 \\
1\end{array}$ & $\begin{array}{r}51 \\
114 \\
129 \\
135 \\
149 \\
125 \\
43 \\
6\end{array}$ & $\begin{array}{l}0.037 \\
0.043 \\
0.040 \\
0.040 \\
0.035 \\
0.037 \\
0.028 \\
0.059\end{array}$ & $\begin{array}{l}0.056 \\
0.058 \\
0.053 \\
0.052 \\
0.056 \\
0.066 \\
0.064 \\
0.060\end{array}$ & $\begin{array}{r}13.2 \\
17.7 \\
15.3 \\
15.7 \\
23.6 \\
18.7 \\
21.8 \\
8.0\end{array}$ & $\begin{array}{l}15 \cdot 1 \\
16 \cdot 3 \\
19.0 \\
20.9 \\
21.9 \\
26 \cdot 2 \\
27 \cdot 7 \\
23.5\end{array}$ \\
\hline over) & - & 15 & - & 159 & 一 & $10 \cdot 600$ & - & 2 & - & $0 \cdot 133$ & - & $79 \cdot 5$ \\
\hline Total & 6,788 & 13,295 & 4,318 & 15,927 & 一 & - & 264 & 754 & - & - & 一 & - \\
\hline
\end{tabular}

TABLE 21: BROAD DIAGNOSTIC GROUP 20: MISCELLANEOUS

(I.S.C. Nos. 000, 240-299, 320-326, 590-617, 750-762, 765-776, 786, 788, 789, 790-795)

\begin{tabular}{|c|c|c|c|c|c|c|c|c|c|c|c|c|}
\hline \multirow[t]{2}{*}{ Age Group } & \multicolumn{2}{|c|}{$\begin{array}{l}\text { Exposure to Risk } \\
\text { (Man Years) }\end{array}$} & \multicolumn{2}{|c|}{$\begin{array}{c}\text { No. of Days } \\
\text { Sickness Absence* }\end{array}$} & \multicolumn{2}{|c|}{$\mid \begin{array}{c}\text { Average Annual } \\
\text { Duration Per Person } \\
\text { (Days) }\end{array}$} & \multicolumn{2}{|c|}{ No. of Spells } & \multicolumn{2}{|c|}{$\begin{array}{c}\text { Annual Inception } \\
\text { Rate } \\
\text { (Spells) }\end{array}$} & \multicolumn{2}{|c|}{$\begin{array}{c}\text { Average Length } \\
\text { of Spell } \\
\text { (Days) }\end{array}$} \\
\hline & Single & Married & Single & Married & Single & Married & Single & Married & Single & Married & Single & Married \\
\hline $\begin{array}{l}\text { Up to } 24 \\
25-29 \\
30-34 \\
35-39 \\
40-44 \\
45-49 \\
50-54 \\
55-59 \\
(60 \text { and }\end{array}$ & $\begin{array}{r}2,100 \\
1,668 \\
1,082 \\
694 \\
625 \\
460 \\
142 \\
17\end{array}$ & $\begin{array}{r}904 \\
1,971 \\
2,447 \\
2,611 \\
2,679 \\
1,898 \\
670 \\
100\end{array}$ & $\begin{array}{r}3,830 \\
3,012 \\
1,470 \\
1,031 \\
825 \\
798 \\
420 \\
15\end{array}$ & $\begin{array}{r}3,221 \\
7,474 \\
6,760 \\
7,303 \\
7,561 \\
4,232 \\
1,842 \\
238\end{array}$ & $\begin{array}{l}1.823 \\
1.804 \\
1.358 \\
1.485 \\
1,320 \\
1.735 \\
2.968 \\
0.882\end{array}$ & $\begin{array}{l}3 \cdot 566 \\
3 \cdot 789 \\
2 \cdot 765 \\
2 \cdot 797 \\
2 \cdot 820 \\
2 \cdot 230 \\
2 \cdot 746 \\
2 \cdot 392\end{array}$ & $\begin{array}{r}312 \\
234 \\
116 \\
65 \\
66 \\
51 \\
12 \\
1\end{array}$ & $\begin{array}{r}241 \\
493 \\
510 \\
481 \\
439 \\
292 \\
103 \\
19\end{array}$ & $\begin{array}{l}0 \cdot 149 \\
0 \cdot 140 \\
0 \cdot 107 \\
0.094 \\
0 \cdot 106 \\
0 \cdot 111 \\
0.085 \\
0.059\end{array}$ & $\begin{array}{l}0 \cdot 267 \\
0 \cdot 250 \\
0 \cdot 209 \\
0 \cdot 184 \\
0 \cdot 164 \\
0 \cdot 154 \\
0 \cdot 154 \\
0 \cdot 191\end{array}$ & $\begin{array}{l}12.3 \\
12.9 \\
12.7 \\
15.9 \\
12.5 \\
15.6 \\
35.0 \\
15.0\end{array}$ & $\begin{array}{l}13.4 \\
15 \cdot 2 \\
13 \cdot 3 \\
15 \cdot 2 \\
17 \cdot 2 \\
14.5 \\
17.9 \\
12.6\end{array}$ \\
\hline over) & - & 15 & - & 15 & - & 1.000 & - & 1 & - & 0.067 & - & $15 \cdot 0$ \\
\hline Total & 6,788 & 13,295 & 11,401 & 38,646 & - & - & 857 & 2,579 & - & - & - & - \\
\hline
\end{tabular}

*Excludes days beyond first six months of absence. 